\title{
Systematic Review \\ Systematic Review and Meta-Analysis of the Effect of Statins on Circulating E-Selectin, L-Selectin, and P-Selectin
}

\author{
Angelo Zinellu ${ }^{1}$ (D) and Arduino A. Mangoni ${ }^{2,3, *(\mathbb{D})}$ \\ 1 Department of Biomedical Sciences, University of Sassari, 07100 Sassari, Italy; azinellu@uniss.it \\ 2 Discipline of Clinical Pharmacology, College of Medicine and Public Health, Flinders University, \\ Bedford Park, SA 5042, Australia \\ 3 Department of Clinical Pharmacology, Flinders Medical Centre, Southern Adelaide Local Health Network, \\ Bedford Park, SA 5042, Australia \\ * Correspondence: arduino.mangoni@flinders.edu.au
}

Citation: Zinellu, A.; Mangoni, A.A. Systematic Review and

Meta-Analysis of the Effect of Statins on Circulating E-Selectin, L-Selectin, and P-Selectin. Biomedicines 2021, 9, 1707. https://doi.org/10.3390/ biomedicines 9111707

Academic Editor: Luca G.

Dalle Carbonare

Received: 1 November 2021

Accepted: 16 November 2021

Published: 17 November 2021

Publisher's Note: MDPI stays neutral with regard to jurisdictional claims in published maps and institutional affiliations.

Copyright: (c) 2021 by the authors. Licensee MDPI, Basel, Switzerland. This article is an open access article distributed under the terms and conditions of the Creative Commons Attribution (CC BY) license (https:/ / creativecommons.org/licenses/by/ $4.0 /)$.

\begin{abstract}
The pleiotropic effects of statins might involve preventing inflammatory cell adhesion to the endothelium, which is a critical step in the pathogenesis of atherosclerosis. We conducted a systematic review and meta-analysis of the effects of statins on the circulating cell adhesion molecules E-Selectin, L-Selectin, and P-Selectin. A literature search was conducted in PubMed, Web of Science, and Scopus, from inception to July 2021. Risk of bias and certainty of evidence were assessed using the Joanna Briggs Institute Critical Appraisal Checklist and GRADE, respectively. In 61 studies, statins significantly reduced P-selectin (standard mean difference, SMD $=-0.39,95 \% \mathrm{CI}-0.55$ to $-0.22, p<0.001$; moderate certainty of evidence), L-selectin (SMD $=-0.49,95 \% \mathrm{CI}-0.89$ to -0.10 , $p=0.014$; very low certainty of evidence), and E-Selectin (SMD $=-0.73,95 \% \mathrm{CI}-1.02$ to -0.43 , $p<0.001$; moderate certainty of evidence), independently of baseline lipid profile and other study and patient characteristics. The corresponding pooled SMD values in sensitivity analysis were not substantially altered when individual studies were sequentially removed. Simvastatin had a significant lowering effect on both P-selectin and E-selectin. Therefore, statins significantly reduce circulating selectins. Further studies are required to investigate whether selectin lowering mediates cardiovascular risk reduction with these agents. (PROSPERO registration number: CRD42021282778).
\end{abstract}

Keywords: statins; E-Selectin; L-Selectin; P-Selectin; atherosclerosis

\section{Introduction}

A critical step in the pathophysiology of atherosclerosis involves the adhesion of inflammatory cell types to the endothelium. This process is facilitated by several cell adhesion molecules [1]. The selectins are a key family of cell adhesion molecules that includes the C-type lectins P-selectin, stored in platelets and endothelial cells [2]; L-selectin, expressed in leukocytes [3]; and E-selectin, expressed in the endothelium [2,4]. L-selectin mediates lymphocyte rolling, whereas P-selectin and E-selectin are primarily expressed in states of endothelial inflammation and facilitate monocyte, neutrophil, and lymphocyte rolling [4]. E-Selectin, L-Selectin, and P-Selectin also exist in soluble forms and can be measured in blood to characterize the state of endothelial and platelet activation in atherosclerosis [4-6].

The pathophysiological role of selectins, particularly P-Selectin and E-selectin, in atherosclerotic cardiovascular disease is supported both by experimental and human studies [7]. In particular, epidemiological studies have reported significant and positive associations between the concentration of soluble selectins and adverse cardiovascular outcomes. For example, higher soluble P-selectin concentrations have been shown to be significantly associated with incident cardiovascular events in women [8]. Similar associations between P-selectin and cardiovascular events have been reported in other studies $[9,10]$. Soluble E-selectin has also shown significant associations with incident cardiovascular disease in patients with renal failure [11] and atrial fibrillation [12]. 
Therefore, the available evidence suggests that soluble selectins play a critical role in the pathophysiology of atherosclerosis and endothelial dysfunction and as markers of cardiovascular risk [7], and that interventions targeting this family of cell adhesion molecules might exert atheroprotective effects [13].

Treatment with statins, the leading class of lipid-lowering agents for cardiovascular prevention $[14,15]$, has been shown to exert beneficial effects on endothelial and vascular homeostasis independently of their primary target, inhibition of 3-hydroxy-3methylglutaryl-CoA (HMG-CoA) reductase [16]. These so-called pleiotropic effects of statins include the suppression of specific pro-inflammatory cytokines with consequent reduced activation of selectins [17-19]. As human studies have investigated the effects of treatment with statins on soluble concentrations of selectins, we sought to critically appraise this evidence by conducting a systematic review and meta-analysis of the effects of statins on circulating E-Selectin, L-Selectin, and P-Selectin. Meta-regression and subgroup analyses were also conducted to investigate associations between effect size and specific study and patient characteristics.

\section{Materials and Methods}

A systematic literature search was conducted in the electronic databases PubMed, Web of Science, and Scopus, from inception to July 2021, using the following terms and their combination: "Selectin" or "P-Selectin" or "L-Selectin" or "E-Selectin" and "Statin". Abstracts were independently screened by two investigators. If relevant, the full-text articles were reviewed according to the following eligibility criteria: (1) assessment of soluble P-Selectin and/or L-Selectin and/or E-Selectin in plasma or serum at baseline and after statin treatment; (2) $\geq 10$ adult participants; (3) English language; and (4) full-text available. The references of the retrieved articles were also searched to identify additional studies. Any disagreement between reviewers was resolved by a third investigator. Data extracted from each study included the year of publication; the continent where the study was conducted; age; the proportion of males; the concentrations of P-Selectin, L-Selectin, and E-Selectin before and after treatment; the primary condition studied; baseline lipid profile; statin and daily dose used; and treatment duration.

The risk of bias was assessed using the Joanna Briggs Institute (JBI) Critical Appraisal Checklist for analytical studies. A score of $\geq 5,4$, and $<4$ indicated low, moderate, and high risk, respectively [20]. The certainty of evidence was assessed using the Grades of Recommendation, Assessment, Development and Evaluation (GRADE) Working Group system. GRADE considers the study design (randomized vs. observational), the risk of bias (JBI checklist), the presence of unexplained heterogeneity, the indirectness of evidence, the imprecision of results (sample size, 95\% confidence interval width and threshold crossing), the effect size (small, SMD < 0.5, moderate, SMD 0.5-0.8, and large, SMD >0.8) [21], and the probability of publication bias [22-24]. The study complied with the Preferred Reporting Items for Systematic reviews and Meta-Analyses (PRISMA) 2020 statement (Supplementary Materials, Tables S1 and S2) [25]. The protocol was registered in the International Prospective Register of Systematic Reviews (PROSPERO registration number: CRD42021282778).

\section{Statistical Analysis}

Standardized mean differences (SMDs) were calculated to generate forest plots of continuous data and to evaluate differences in selectin serum concentrations before and after statin treatment. If necessary, means and standard deviations were extrapolated from medians and interquartile ranges [26], or from graphs using the Graph Data Extractor software. Heterogeneity of SMD across studies was assessed using the Q-statistic (significance level set at $p<0.10)$ and $\mathrm{I}^{2}$-statistic $(<25 \%$, no heterogeneity; between $25-50 \%$, moderate heterogeneity; between $50-75 \%$, large heterogeneity; and $>75 \%$, extreme heterogeneity) $[27,28]$. Random-effects models were used in the presence of significant heterogeneity $\left(\mathrm{I}^{2} \geq 50 \%\right)$. Sensitivity analysis was conducted to investigate the influence of each study on the over- 
all risk estimate [29]. The presence of publication bias was assessed using the Begg's and Egger's tests (significance level set at $p<0.05$ ) [30,31], and the Duval and Tweedie "trim-and-fill" method [32].

Univariate meta-regression analyses were conducted to investigate associations between effect size and the following study and patient characteristics: age; proportion of males; body mass index; baseline total cholesterol, low-density lipoproteins (LDL)cholesterol, high-density lipoproteins (HDL)-cholesterol, and triglycerides; year of publication; sample size; continent where the study was conducted; specific statin and class used (lipophilic: atorvastatin, simvastatin, lovastatin, fluvastatin, cerivastatin, and pitavastatin; hydrophilic: rosuvastatin, pravastatin); and treatment duration. Pre-planned subgroup analyses investigated the effects of specific statins, statin classes, and continent where the study was conducted. Statistical analyses were performed using Stata 14 (STATA Corp., College Station, TX, USA).

\section{Results}

\subsection{Systematic Research}

A flow chart describing the screening process is presented in Figure 1. We initially identified 1698 articles. A total of 1613 were excluded after the first screening because they were either duplicates or irrelevant. After a full-text review of the remaining 85 articles, 24 were further excluded due to missing data $(n=7)$ or because they did not fulfil the inclusion criteria $(\mathrm{n}=1$, age $<18$ years; $\mathrm{n}=4$, participants already on lipid-lowering treatment; $\mathrm{n}=3$, sample size $<10 ; \mathrm{n}=9$ measurement of cell surface selectin). Thus, 61 studies published between 1999 and 2018 were included in the final analysis (Table 1) [33-93].

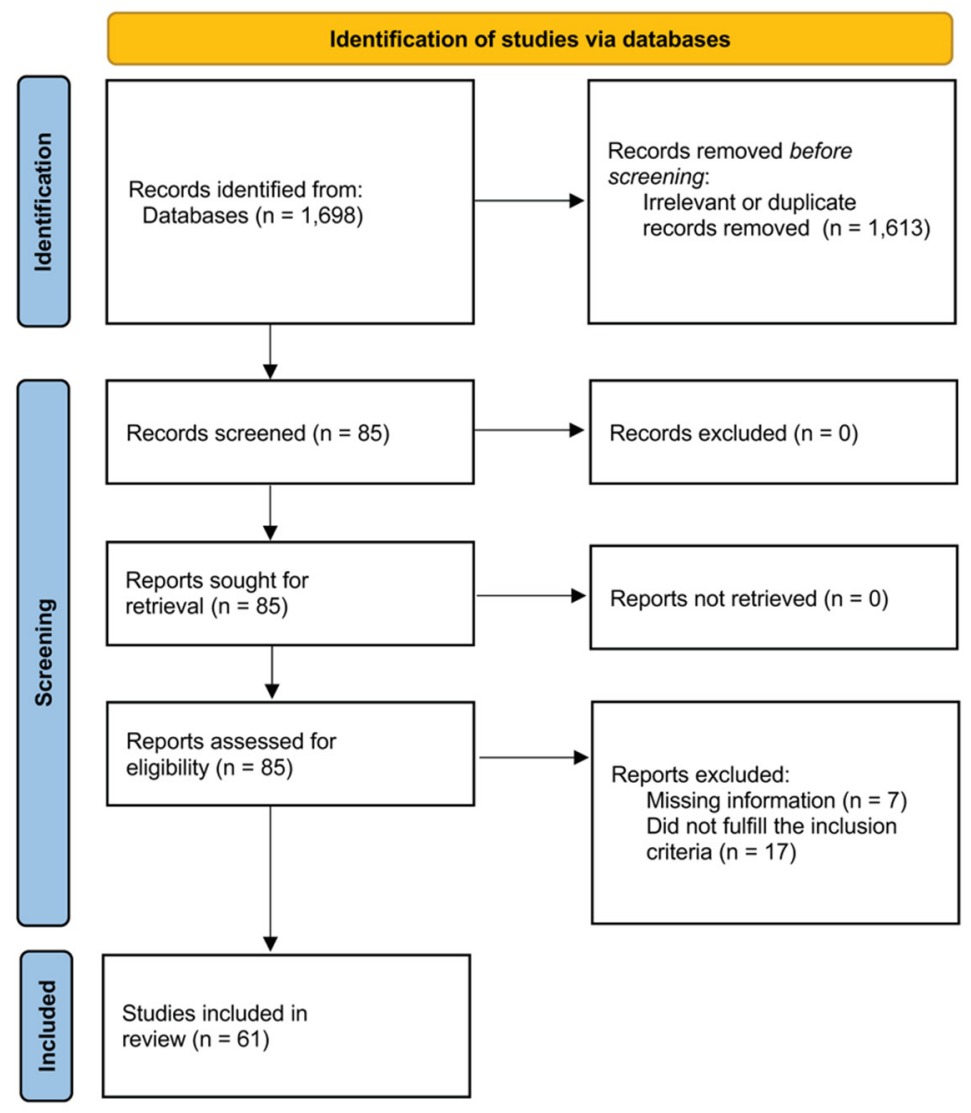

Figure 1. PRISMA 2020 flow chart. 
Table 1. Study characteristics.

\begin{tabular}{|c|c|c|c|c|c|c|c|c|c|c|}
\hline First Author, Year, Country [Reference] & $\mathbf{N}$ & $\begin{array}{l}\text { Age } \\
\text { (Years) }\end{array}$ & $\begin{array}{c}\text { Males } \\
(\%)\end{array}$ & $\begin{array}{l}\text { P-Selectinbefore } \\
\text { Mean } \pm \text { SD } \\
\text { (ng/mL) }\end{array}$ & $\begin{array}{l}\text { P-Selectinafter } \\
\text { Mean } \pm \text { SD } \\
(\mathrm{ng} / \mathrm{mL})\end{array}$ & $\begin{array}{l}\text { L-Selectinbefore } \\
\text { Mean } \pm \text { SD } \\
\text { (ng/mL) }\end{array}$ & $\begin{array}{l}\text { L-Selectinafter } \\
\text { Mean } \pm \text { SD } \\
\text { (ng/mL) }\end{array}$ & $\begin{array}{l}\text { E-Selectinbefore } \\
\text { Mean } \pm \text { SD } \\
\text { (ng/mL) }\end{array}$ & $\begin{array}{l}\text { E-Selectinafter } \\
\text { Mean } \pm \text { SD } \\
\text { (ng/mL) }\end{array}$ & $\begin{array}{l}\text { Primary Condition } \\
\text { Statin and Daily Dose } \\
\text { Treatment Duration }\end{array}$ \\
\hline Koh KK, 1999, USA [33] & 28 & 57 & 0 & - & - & - & - & $49 \pm 18$ & $51 \pm 19$ & $\begin{array}{l}\text { Hypercholesterolaemia } \\
\text { Simvastatin } 10 \mathrm{mg} \\
6 \text { weeks }\end{array}$ \\
\hline Rauch U (a), 2000, USA [34] & 24 & 62 & NR & $130 \pm 30$ & $129 \pm 22$ & $753 \pm 30$ & $738 \pm 31$ & - & - & $\begin{array}{l}\text { Hypercholesterolaemia } \\
\text { Pravastatin } 40 \mathrm{mg} \\
12 \text { weeks }\end{array}$ \\
\hline Rauch U (b), 2000, USA [34] & 26 & 58 & NR & $105 \pm 17$ & $115 \pm 15$ & $834 \pm 42$ & $778 \pm 32$ & - & - & $\begin{array}{l}\text { Hypercholesterolaemia } \\
\text { Simvastatin } 20 \mathrm{mg} \\
12 \text { weeks }\end{array}$ \\
\hline Romano M, 2000, Italy [35] & 13 & 59 & 23 & $118 \pm 63$ & $81 \pm 36$ & - & - & - & - & $\begin{array}{l}\text { Hypercholesterolaemia } \\
\text { Fluvastatin } 80 \mathrm{mg} \\
12 \text { weeks } \\
\end{array}$ \\
\hline Sbarouni E, 2000, Greece [36] & 16 & 66 & 0 & - & - & - & - & $17 \pm 28$ & $17 \pm 30$ & $\begin{array}{c}\text { Ischaemic heart disease } \\
\text { Simvastatin } 20 \mathrm{mg} \\
8 \text { weeks }\end{array}$ \\
\hline Alonso R, 2001, Spain [37] & 25 & 48 & 44 & - & - & - & - & $16 \pm 3$ & $18 \pm 4$ & $\begin{array}{c}\text { Familial } \\
\text { hypercholesterolaemia } \\
\text { Simvastatin } 40-80 \mathrm{mg} \\
52 \text { weeks } \\
\end{array}$ \\
\hline Blann AD, 2001, UK [38] & 17 & 65 & 59 & $30 \pm 9$ & $26 \pm 10$ & - & - & - & - & $\begin{array}{l}\text { Peripheral artery disease } \\
\text { Pravastatin } 40 \mathrm{mg} \\
\text { 4 weeks }\end{array}$ \\
\hline Sardo MA, 2001, Italy [39] & 20 & 45 & 45 & - & - & - & - & $46 \pm 12$ & $51 \pm 17$ & $\begin{array}{l}\text { Hypercholesterolaemia } \\
\text { Simvastatin } 20 \mathrm{mg} \\
24 \text { weeks }\end{array}$ \\
\hline Solheim S, 2001, Norway [40] & 40 & 54 & 100 & $54 \pm 13$ & $52 \pm 13$ & - & - & $36 \pm 8$ & $34 \pm 8$ & $\begin{array}{l}\text { Hypercholesterolaemia } \\
\text { Pravastatin } 40 \mathrm{mg} \\
8 \text { weeks } \\
\end{array}$ \\
\hline van Haelst PL, 2001, The Netherlands [41] & 10 & 52 & 90 & - & - & - & - & $59 \pm 12$ & $45 \pm 12$ & $\begin{array}{c}\text { Ischaemic heart disease } \\
\text { Fluvastatin } 80 \mathrm{mg} \\
48 \text { weeks }\end{array}$ \\
\hline Atalar E, 2002, Turkey [42] & 36 & 53 & 61 & - & - & $666 \pm 201$ & $584 \pm 162$ & - & - & $\begin{array}{l}\text { Hypercholesterolaemia } \\
\text { Atorvastatin } 10 \mathrm{mg} \\
12 \text { weeks }\end{array}$ \\
\hline
\end{tabular}


Table 1. Cont.

\begin{tabular}{|c|c|c|c|c|c|c|c|c|c|c|}
\hline First Author, Year, Country [Reference] & $\mathbf{N}$ & $\begin{array}{l}\text { Age } \\
\text { (Years) }\end{array}$ & $\begin{array}{c}\text { Males } \\
(\%)\end{array}$ & $\begin{array}{l}\text { P-Selectinbefore } \\
\text { Mean } \pm \text { SD } \\
\text { (ng/mL) }\end{array}$ & $\begin{array}{l}\text { P-Selectinafter } \\
\text { Mean } \pm \text { SD } \\
\text { (ng/mL) }\end{array}$ & $\begin{array}{l}\text { L-Selectinbefore } \\
\text { Mean } \pm \text { SD } \\
\text { (ng/mL) }\end{array}$ & $\begin{array}{l}\text { L-Selectinafter } \\
\text { Mean } \pm \text { SD } \\
(\mathrm{ng} / \mathrm{mL})\end{array}$ & $\begin{array}{l}\text { E-Selectinbefore } \\
\text { Mean } \pm \text { SD } \\
\text { (ng/mL) }\end{array}$ & $\begin{array}{l}\text { E-Selectinafter } \\
\text { Mean } \pm \text { SD } \\
(\mathrm{ng} / \mathrm{mL})\end{array}$ & $\begin{array}{l}\text { Primary Condition } \\
\text { Statin and Daily Dose } \\
\text { Treatment Duration }\end{array}$ \\
\hline Seljeflot I (a), 2002, Norway [43] & 28 & NR & 79 & $99 \pm 30$ & $86 \pm 21$ & - & - & $43 \pm 19$ & $42 \pm 19$ & $\begin{array}{c}\text { Ischaemic heart disease } \\
\text { Atorvastatin } 20 \mathrm{mg} \\
12 \text { weeks }\end{array}$ \\
\hline Seljeflot I (b), 2002, Norway [43] & 30 & NR & 93 & $106 \pm 30$ & $96 \pm 36$ & - & - & $49 \pm 19$ & $47 \pm 21$ & $\begin{array}{l}\text { Ischaemic heart disease } \\
\text { Simvastatin } 20 \mathrm{mg} \\
12 \text { weeks }\end{array}$ \\
\hline Bogaty P, 2003, Canada [44] & 17 & 60 & 6 & $56 \pm 20$ & $58 \pm 23$ & - & - & $74 \pm 18$ & $54 \pm 14$ & $\begin{array}{c}\text { Ischaemic heart disease } \\
\text { Atorvastatin } 10-80 \mathrm{mg} \\
11 \text { weeks }\end{array}$ \\
\hline Dalla Nora I, 2003, Italy [45] & 13 & 66 & 54 & - & - & - & - & $16 \pm 3$ & $8 \pm 1$ & $\begin{array}{c}\text { Type } 2 \text { diabetes } \\
\text { Atorvastatin } 10 \mathrm{mg} \\
12 \text { weeks }\end{array}$ \\
\hline Empen K, 2003, Germany [46] & 11 & 62 & 55 & - & - & - & - & $70 \pm 37$ & $67 \pm 40$ & $\begin{array}{c}\text { Type } 2 \text { diabetes } \\
\text { Atorvastatin } 10 \mathrm{mg} \\
6 \text { weeks }\end{array}$ \\
\hline Ferroni P, 2003, Italy [47] & 25 & 54 & 36 & $70 \pm 20$ & $52 \pm 18$ & - & - & - & - & $\begin{array}{l}\text { Hypercholesterolaemia } \\
\text { Simvastatin } 20 \mathrm{mg} \\
8 \text { weeks }\end{array}$ \\
\hline Hernández C, 2003, Spain [48] & 44 & 50 & 100 & - & - & - & - & $36 \pm 4$ & $33 \pm 4$ & $\begin{array}{c}\text { Hypercholesterolaemia } \\
\text { Various statins and doses } \\
16 \text { weeks }\end{array}$ \\
\hline Homma Y, 2003, Japan [49] & 30 & 67 & 13 & $250 \pm 103$ & $196 \pm 81$ & - & - & - & - & $\begin{array}{c}\text { Type } 2 \\
\text { hyperlipoproteinemia } \\
\text { Fluvastatin } 20-40 \mathrm{mg} \\
24 \text { weeks }\end{array}$ \\
\hline Malyszko J, 2003, Poland [50] & 12 & NR & 58 & $115 \pm 52$ & $84 \pm 13$ & - & - & - & - & $\begin{array}{c}\text { Kidney transplant } \\
\text { Fluvastatin } 20 \mathrm{mg} \\
12 \text { weeks }\end{array}$ \\
\hline Nawawi H (a), 2003, Malaysia [51] & 27 & 42 & 41 & - & - & - & - & $209 \pm 18$ & $35 \pm 11$ & $\begin{array}{c}\text { Familial } \\
\text { hypercholesterolaemia } \\
\text { Atorvastatin } 80 \mathrm{mg} \\
9 \text { weeks }\end{array}$ \\
\hline Nawawi H (b), 2003, Malaysia [51] & 47 & 48 & 55 & - & - & - & - & $246 \pm 14$ & $144 \pm 11$ & $\begin{array}{c}\text { Familial } \\
\text { hypercholesterolaemia } \\
\text { Atorvastatin } 10 \mathrm{mg} \\
9 \text { weeks }\end{array}$ \\
\hline
\end{tabular}


Table 1. Cont.

\begin{tabular}{|c|c|c|c|c|c|c|c|c|c|c|}
\hline First Author, Year, Country [Reference] & $\mathbf{N}$ & $\begin{array}{l}\text { Age } \\
\text { (Years) }\end{array}$ & $\begin{array}{c}\text { Males } \\
(\%)\end{array}$ & $\begin{array}{l}\text { P-Selectinbefore } \\
\text { Mean } \pm \text { SD } \\
\text { (ng/mL) }\end{array}$ & $\begin{array}{l}\text { P-Selectinafter } \\
\text { Mean } \pm \text { SD } \\
\text { (ng/mL) }\end{array}$ & $\begin{array}{l}\text { L-Selectinbefore } \\
\text { Mean } \pm \text { SD } \\
\text { (ng/mL) }\end{array}$ & $\begin{array}{l}\text { L-Selectinafter } \\
\text { Mean } \pm \text { SD } \\
(\mathrm{ng} / \mathrm{mL})\end{array}$ & $\begin{array}{l}\text { E-Selectinbefore } \\
\text { Mean } \pm \text { SD } \\
\text { (ng/mL) }\end{array}$ & $\begin{array}{c}\text { E-Selectinafter } \\
\text { Mean } \pm \text { SD } \\
(\mathrm{ng} / \mathrm{mL})\end{array}$ & $\begin{array}{l}\text { Primary Condition } \\
\text { Statin and Daily Dose } \\
\text { Treatment Duration }\end{array}$ \\
\hline Puccetti L, 2003, Italy [52] & 14 & 50 & 57 & $23 \pm 4$ & $10 \pm 2$ & - & - & - & - & $\begin{array}{l}\text { Hypercholesterolaemia } \\
\text { Simvastatin } 20 \mathrm{mg} \\
6 \text { weeks }\end{array}$ \\
\hline van Haelst PL, 2003, The Netherlands [53] & 35 & 42 & 60 & - & - & - & - & $69 \pm 27$ & $62 \pm 27$ & $\begin{array}{c}\text { Familial } \\
\text { hypercholesterolaemia } \\
\text { Simvastatin } 80 \mathrm{mg} \\
52 \text { weeks }\end{array}$ \\
\hline Brown SL, 2004, South Africa [54] & 23 & 36 & 70 & - & - & - & - & $61 \pm 58$ & $61 \pm 55$ & $\begin{array}{c}\text { Familial } \\
\text { hypercholesterolaemia } \\
\text { Simvastatin } 20-80 \mathrm{mg} \\
24 \text { weeks }\end{array}$ \\
\hline Ceriello A, 2004, Italy [55] & 30 & 54 & 73 & - & - & - & - & $79 \pm 73$ & $46 \pm 55$ & $\begin{array}{c}\text { Type } 2 \text { diabetes } \\
\text { Simvastatin } 40 \mathrm{mg} \\
12 \text { weeks }\end{array}$ \\
\hline Cha JK, 2004, Korea [56] & 32 & 60 & 87 & $97 \pm 12$ & $87 \pm 8$ & - & - & - & - & $\begin{array}{c}\text { Ischaemic stroke } \\
\text { Simvastatin } 20 \mathrm{mg} \\
12 \text { weeks }\end{array}$ \\
\hline Malyszko J, 2004, Poland [58] & 15 & 50 & NR & $103 \pm 46$ & $94 \pm 36$ & - & - & $64 \pm 26$ & $51 \pm 10$ & $\begin{array}{c}\text { Peritoneal dialysis } \\
\text { Simvastatin } 10 \mathrm{mg} \\
24 \text { weeks }\end{array}$ \\
\hline Nomura S (a), 2004, Japan [59] & 30 & 67 & 37 & $190 \pm 23$ & $164 \pm 23$ & - & - & - & - & $\begin{array}{l}\text { Hypertension and } \\
\text { diabetes } \\
\text { Simvastatin } 10 \mathrm{mg} \\
24 \text { weeks }\end{array}$ \\
\hline Nomura S (b), 2004, Japan [59] & 18 & 64 & 56 & $108 \pm 15$ & $107 \pm 15$ & - & - & - & - & $\begin{array}{c}\text { Hypertension } \\
\text { Simvastatin } 10 \mathrm{mg} \\
24 \text { weeks }\end{array}$ \\
\hline Skhra J, 2004, Czech Republic [60] & 20 & 57 & 60 & $190 \pm 54$ & $199 \pm 55$ & - & - & $65 \pm 19$ & $70 \pm 19$ & $\begin{array}{c}\text { Type } 2 \text { diabetes } \\
\text { Simvastatin } 20 \mathrm{mg} \\
12 \text { weeks }\end{array}$ \\
\hline Akçay MN, 2005, Turkey [61] & 10 & 41 & 40 & - & - & - & - & $24 \pm 3$ & $11 \pm 1$ & $\begin{array}{c}\text { Type } 2 \text { diabetes } \\
\text { Simvastatin } 20 \mathrm{mg} \\
12 \text { weeks }\end{array}$ \\
\hline
\end{tabular}


Table 1. Cont.

\begin{tabular}{|c|c|c|c|c|c|c|c|c|c|c|}
\hline First Author, Year, Country [Reference] & $\mathbf{N}$ & $\begin{array}{l}\text { Age } \\
\text { (Years) }\end{array}$ & $\begin{array}{c}\text { Males } \\
(\%)\end{array}$ & $\begin{array}{l}\text { P-Selectinbefore } \\
\text { Mean } \pm \text { SD } \\
(\mathrm{ng} / \mathrm{mL})\end{array}$ & $\begin{array}{l}\text { P-Selectinafter } \\
\text { Mean } \pm \text { SD } \\
(\mathrm{ng} / \mathrm{mL})\end{array}$ & $\begin{array}{l}\text { L-Selectinbefore } \\
\text { Mean } \pm \text { SD } \\
(\mathrm{ng} / \mathrm{mL})\end{array}$ & $\begin{array}{l}\text { L-Selectinafter } \\
\text { Mean } \pm \text { SD } \\
\text { (ng/mL) }\end{array}$ & $\begin{array}{l}\text { E-Selectinbefore } \\
\text { Mean } \pm \text { SD } \\
(\mathrm{ng} / \mathrm{mL})\end{array}$ & $\begin{array}{l}\text { E-Selectinafter } \\
\text { Mean } \pm \text { SD } \\
\text { (ng/mL) }\end{array}$ & $\begin{array}{l}\text { Primary Condition } \\
\text { Statin and Daily Dose } \\
\text { Treatment Duration }\end{array}$ \\
\hline Undas A, 2005, Poland [62] & 20 & 56 & 70 & $118 \pm 54$ & $100 \pm 45$ & - & - & - & - & $\begin{array}{l}\text { Hypercholesterolaemia } \\
\text { Simvastatin } 40 \mathrm{mg} \\
4 \text { weeks }\end{array}$ \\
\hline Bleske BE, 2006, USA [63] & 15 & 56 & 60 & $30 \pm 19$ & $23 \pm 20$ & - & - & - & - & $\begin{array}{c}\text { Non-ischaemic } \\
\text { cardiomyopathy } \\
\text { Atorvastatin } 80 \mathrm{mg} \\
12 \text { weeks }\end{array}$ \\
\hline Marschang P, 2006, Austria [64] & 47 & 59 & 64 & $92 \pm 75$ & $52 \pm 27$ & - & - & $55 \pm 27$ & $60 \pm 27$ & $\begin{array}{c}\text { Ischaemic heart disease } \\
\text { Various statins and doses } \\
12 \text { weeks }\end{array}$ \\
\hline Peverill RE, 2006, Australia [65] & 24 & 59 & 0 & $43 \pm 11$ & $34 \pm 9$ & - & - & - & - & $\begin{array}{l}\text { Hypercholesterolaemia } \\
\text { Pravastatin } 20 \mathrm{mg} \\
24 \text { weeks }\end{array}$ \\
\hline Potaczek PD (a), 2006, Poland [66] & 10 & 54 & NR & $135 \pm 63$ & $129 \pm 66$ & - & - & - & - & $\begin{array}{c}\text { Hypercholesterolaemia } \\
\text { Simvastatin } 40 \mathrm{mg} \\
4 \text { weeks }\end{array}$ \\
\hline Alber HF, 2007, Austria [67] & 15 & 57 & NR & - & - & - & - & $56 \pm 23$ & $52 \pm 25$ & $\begin{array}{c}\text { Ischaemic heart disease } \\
\text { Atorvastatin } 20 \mathrm{mg} \\
12 \text { weeks }\end{array}$ \\
\hline Inami N (a), 2007, Japan [68] & 65 & 65 & 35 & $177 \pm 226$ & $203 \pm 282$ & - & - & - & - & $\begin{array}{l}\text { Hypercholesterolaemia } \\
\text { without diabetes } \\
\text { Pitavastatin } 2 \mathrm{mg} \\
24 \text { weeks }\end{array}$ \\
\hline Inami N (b), 2007, Japan [68] & 52 & 62 & 46 & $238 \pm 260$ & $200 \pm 296$ & - & - & - & - & $\begin{array}{l}\text { Hypercholesterolaemia } \\
\text { and diabetes } \\
\text { Pitavastatin } 2 \mathrm{mg} \\
24 \text { weeks }\end{array}$ \\
\hline Jeffs LS, 2007, Australia [69] & 25 & 57 & 64 & - & - & - & - & $3.6 \pm 1.5$ & $3.9 \pm 1.6$ & $\begin{array}{c}\text { End-stage renal disease } \\
\text { Pravastatin } 10-40 \mathrm{mg} \\
20 \text { weeks }\end{array}$ \\
\hline Souza-Costa DC (a), 2007, Brazil [70] & 15 & 28 & 100 & $30 \pm 16$ & $29 \pm 15$ & - & - & - & - & $\begin{array}{c}\text { Healthy } \\
\text { Atorvastatin } 10 \mathrm{mg} \\
2 \text { weeks }\end{array}$ \\
\hline
\end{tabular}


Table 1. Cont.

\begin{tabular}{|c|c|c|c|c|c|c|c|c|c|c|}
\hline First Author, Year, Country [Reference] & $\mathbf{N}$ & $\begin{array}{l}\text { Age } \\
\text { (Years) }\end{array}$ & $\begin{array}{c}\text { Males } \\
(\%)\end{array}$ & $\begin{array}{l}\text { P-Selectinbefore } \\
\text { Mean } \pm \text { SD } \\
(\mathrm{ng} / \mathrm{mL})\end{array}$ & $\begin{array}{l}\text { P-Selectinafter } \\
\text { Mean } \pm \text { SD } \\
(\mathrm{ng} / \mathrm{mL})\end{array}$ & $\begin{array}{l}\text { L-Selectinbefore } \\
\text { Mean } \pm \text { SD } \\
(\mathrm{ng} / \mathrm{mL})\end{array}$ & $\begin{array}{l}\text { L-Selectinafter } \\
\text { Mean } \pm \text { SD } \\
(\mathrm{ng} / \mathrm{mL})\end{array}$ & $\begin{array}{l}\text { E-Selectinbefore } \\
\text { Mean } \pm \text { SD } \\
\text { (ng/mL) }\end{array}$ & $\begin{array}{l}\text { E-Selectinafter } \\
\text { Mean } \pm \text { SD } \\
(\mathrm{ng} / \mathrm{mL})\end{array}$ & $\begin{array}{l}\text { Primary Condition } \\
\text { Statin and Daily Dose } \\
\text { Treatment Duration }\end{array}$ \\
\hline Souza-Costa DC (b), 2007, Brazil [70] & 15 & 31 & 100 & $42 \pm 18$ & $31 \pm 21$ & - & - & - & - & $\begin{array}{l}\text { Healthy } \\
\text { Atorvastatin } 10 \mathrm{mg} \\
2 \text { weeks }\end{array}$ \\
\hline Barreto AC, 2008, Brazil [71] & 30 & 35 & 40 & $40 \pm 22$ & $35 \pm 16$ & - & - & - & - & $\begin{array}{l}\text { Pulmonary arterial } \\
\text { hypertension } \\
\text { Rosuvastatin } 10 \mathrm{mg} \\
24 \text { weeks }\end{array}$ \\
\hline Bolewski A, 2008, France [72] & 26 & 57 & 62 & $55 \pm 26$ & $54 \pm 20$ & - & - & $36 \pm 20$ & $38 \pm 20$ & $\begin{array}{l}\text { Hypercholesterolaemia } \\
\text { Atorvastatin } 20 \mathrm{mg} \\
12 \text { weeks }\end{array}$ \\
\hline Del Papa N, 2008, Italy [73] & 20 & 59 & 0 & - & - & - & - & $52 \pm 89$ & $38 \pm 45$ & $\begin{array}{c}\text { Systemic sclerosis } \\
\text { Simvastatin } 20 \mathrm{mg} \\
12 \text { weeks }\end{array}$ \\
\hline Forst T, 2008, Germany [74] & 80 & 62 & 48 & $277 \pm 125$ & $291 \pm 161$ & - & - & - & - & $\begin{array}{c}\text { High cardiovascular risk } \\
\text { Atorvastatin } 40 \mathrm{mg} \\
24 \text { weeks }\end{array}$ \\
\hline Nomura S (a), 2008, Japan [76] & 30 & 60 & 43 & $136 \pm 33$ & $128 \pm 62$ & $784 \pm 121$ & $769 \pm 114$ & $52 \pm 10$ & $42 \pm 11$ & $\begin{array}{l}\text { Hypercholesterolaemia } \\
\text { Pitavastatin } 2 \mathrm{mg} \\
24 \text { weeks }\end{array}$ \\
\hline Nomura S (b), 2008, Japan [76] & 45 & 62 & 44 & $238 \pm 64$ & $223 \pm 33$ & $896 \pm 141$ & $814 \pm 129$ & $74 \pm 21$ & $51 \pm 10$ & $\begin{array}{l}\text { Hypercholesterolaemia } \\
\text { Pitavastatin } 2 \mathrm{mg} \\
24 \text { weeks }\end{array}$ \\
\hline Stulc T, 2008, Czech Republic [77] & 27 & 52 & 30 & - & - & - & - & $58 \pm 38$ & $59 \pm 35$ & $\begin{array}{l}\text { Hypercholesterolemia } \\
\text { Atorvastatin } 20 \mathrm{mg} \\
12 \text { weeks }\end{array}$ \\
\hline Wang L, 2008, USA [78] & 25 & NR & NR & $111 \pm 36$ & $119 \pm 71$ & - & - & - & - & $\begin{array}{l}\text { Metabolic syndrome } \\
\text { Simvastatin } 40 \mathrm{mg} \\
8 \text { weeks }\end{array}$ \\
\hline Baldassarre D, 2009, Italy [79] & 85 & 58 & 85 & - & - & - & - & $32 \pm 15$ & $22 \pm 10$ & $\begin{array}{c}\text { Ischaemic heart disease } \\
\text { Atorvastatin } 20 \mathrm{mg} \\
12 \text { weeks }\end{array}$ \\
\hline
\end{tabular}


Table 1. Cont.

\begin{tabular}{|c|c|c|c|c|c|c|c|c|c|c|}
\hline First Author, Year, Country [Reference] & $\mathbf{N}$ & $\begin{array}{l}\text { Age } \\
\text { (Years) }\end{array}$ & $\begin{array}{c}\text { Males } \\
(\%)\end{array}$ & $\begin{array}{l}\text { P-Selectinbefore } \\
\text { Mean } \pm \text { SD } \\
\text { (ng/mL) }\end{array}$ & $\begin{array}{l}\text { P-Selectinafter } \\
\text { Mean } \pm \text { SD } \\
\text { (ng/mL) }\end{array}$ & $\begin{array}{l}\text { L-Selectinbefore } \\
\text { Mean } \pm \text { SD } \\
(\mathrm{ng} / \mathrm{mL})\end{array}$ & $\begin{array}{l}\text { L-Selectinafter } \\
\text { Mean } \pm \text { SD } \\
\text { (ng/mL) }\end{array}$ & $\begin{array}{l}\text { E-Selectinbefore } \\
\text { Mean } \pm \text { SD } \\
\text { (ng/mL) }\end{array}$ & $\begin{array}{l}\text { E-Selectinafter } \\
\text { Mean } \pm \text { SD } \\
(\mathrm{ng} / \mathrm{mL})\end{array}$ & $\begin{array}{l}\text { Primary Condition } \\
\text { Statin and Daily Dose } \\
\text { Treatment Duration }\end{array}$ \\
\hline Grip O, 2009, Sweden [80] & 10 & 32 & 50 & $255 \pm 105$ & $222 \pm 41$ & - & - & $18 \pm 9$ & $15 \pm 9$ & $\begin{array}{c}\text { Crohn's disease } \\
\text { Atorvastatin } 80 \mathrm{mg} \\
12 \text { weeks }\end{array}$ \\
\hline Nomura S (a), 2009, Japan [81] & 63 & 61 & NR & $188 \pm 50$ & $182 \pm 48$ & - & - & - & - & $\begin{array}{l}\text { Hypercholesterolaemia } \\
\text { Simvastatin } 10 \mathrm{mg} \\
24 \text { weeks }\end{array}$ \\
\hline Nomura S (b), 2009, Japan [81] & 72 & 61 & NR & $184 \pm 47$ & $175 \pm 51$ & - & - & - & - & $\begin{array}{l}\text { Hypercholesterolaemia } \\
\text { Pitavastatin } 2 \mathrm{mg} \\
24 \text { weeks }\end{array}$ \\
\hline Prázný M, 2009, Czech Republic [82] & 20 & 57 & 50 & $203 \pm 64$ & $178 \pm 51$ & - & - & $66 \pm 27$ & $33 \pm 10$ & $\begin{array}{c}\text { Type } 2 \text { diabetes } \\
\text { Simvastatin } 20 \mathrm{mg} \\
12 \text { weeks }\end{array}$ \\
\hline Serrano CV, 2009, Brazil [83] & 23 & 63 & 56 & - & - & - & - & $46 \pm 17$ & $59 \pm 24$ & $\begin{array}{l}\text { Hypercholesterolaemia } \\
\text { Simvastatin } 40 \mathrm{mg} \\
12 \text { weeks }\end{array}$ \\
\hline Fichtenbaum CJ, 2010, USA [84] & 37 & NR & 92 & $61 \pm 21$ & $57 \pm 13$ & - & - & - & - & $\begin{array}{l}\text { Hypercholesterolaemia } \\
\text { Pravastatin } 40 \mathrm{mg} \\
12 \text { weeks }\end{array}$ \\
\hline Kater AL, 2010, Brazil [85] & 25 & 53 & 76 & - & - & - & - & $44 \pm 20$ & $40 \pm 15$ & $\begin{array}{c}\text { Pre-diabetes } \\
\text { Simvastatin } 20 \mathrm{mg} \\
12 \text { weeks }\end{array}$ \\
\hline Kirmizis K, 2010, Greece [86] & 25 & 63 & 48 & - & - & - & - & $77 \pm 31$ & $69 \pm 31$ & $\begin{array}{c}\text { Haemodialysis } \\
\text { Simvastatin } 10 \mathrm{mg} \\
24 \text { weeks }\end{array}$ \\
\hline Xu DY, 2010, China [87] & 79 & 64 & 59 & $101 \pm 19$ & $73 \pm 15$ & - & - & - & - & $\begin{array}{c}\text { High cardiovascular risk } \\
\text { Atorvastatin } 10 \mathrm{mg} \\
8 \text { weeks }\end{array}$ \\
\hline Wu YW, 2012, Taiwan [88] & 34 & 54 & 71 & - & - & - & - & $44 \pm 50$ & $42 \pm 50$ & $\begin{array}{l}\text { Ischaemic heart disease } \\
\text { Atorvastatin } 40 \mathrm{mg} \\
12 \text { weeks }\end{array}$ \\
\hline Altun I, 2014, Turkey [89] & 30 & 53 & 100 & - & - & - & - & $100 \pm 35$ & $89 \pm 31$ & $\begin{array}{l}\text { Acute coronary } \\
\text { syndrome } \\
\text { Atorvastatin } 40 \mathrm{mg} \\
12 \text { weeks }\end{array}$ \\
\hline
\end{tabular}


Table 1. Cont.

\begin{tabular}{|c|c|c|c|c|c|c|c|c|c|c|}
\hline First Author, Year, Country [Reference] & $\mathbf{N}$ & $\begin{array}{c}\text { Age } \\
\text { (Years) }\end{array}$ & $\begin{array}{c}\text { Males } \\
(\%)\end{array}$ & $\begin{array}{l}\text { P-Selectinbefore } \\
\text { Mean } \pm \text { SD } \\
\text { (ng/mL) }\end{array}$ & $\begin{array}{l}\text { P-Selectinafter } \\
\text { Mean } \pm \text { SD } \\
\text { (ng/mL) }\end{array}$ & $\begin{array}{l}\text { L-Selectinbefore } \\
\text { Mean } \pm \text { SD } \\
\text { (ng/mL) }\end{array}$ & $\begin{array}{l}\text { L-Selectinafter } \\
\text { Mean } \pm \text { SD } \\
(\mathrm{ng} / \mathrm{mL})\end{array}$ & $\begin{array}{l}\text { E-Selectinbefore } \\
\text { Mean } \pm \text { SD } \\
\text { (ng/mL) }\end{array}$ & $\begin{array}{l}\text { E-Selectinafter } \\
\text { Mean } \pm \text { SD } \\
\text { (ng/mL) }\end{array}$ & $\begin{array}{l}\text { Primary Condition } \\
\text { Statin and Daily Dose } \\
\text { Treatment Duration }\end{array}$ \\
\hline Pawelczyk M, 2015, Poland [90] & 31 & 62 & 57 & $124 \pm 60$ & $78 \pm 38$ & - & - & - & - & $\begin{array}{l}\text { Ischaemic stroke, } \\
\text { Simvastatin } 20 \mathrm{mg} \\
24 \text { weeks }\end{array}$ \\
\hline Hernandez-Mijares A, 2016, Spain [91] & 20 & 58 & 33 & - & - & - & - & $45 \pm 31$ & $39 \pm 20$ & $\begin{array}{c}\text { Hypercholesterolaemia, } \\
\text { Simvastatin } 40 \mathrm{mg} \\
4 \text { weeks }\end{array}$ \\
\hline Barale C, 2018, Italy [92] & 25 & 59 & 44 & - & - & - & - & $44 \pm 15$ & $25 \pm 8$ & $\begin{array}{l}\text { Hypercholesterolaemia } \\
\text { Simvastatin } 40 \mathrm{mg} \\
8 \text { weeks }\end{array}$ \\
\hline Kotyla PJ, 2018, Poland [93] & 25 & 55 & 12 & $67 \pm 41$ & $66 \pm 26$ & $887 \pm 222$ & $927 \pm 385$ & $276 \pm 122$ & $253 \pm 125$ & $\begin{array}{c}\text { Systemic sclerosis } \\
\text { Simvastatin } 20 \mathrm{mg} \\
4 \text { weeks }\end{array}$ \\
\hline
\end{tabular}

Legend: NR, not reported. 
3.2. Meta-Analysis of Soluble P-Selectin

3.2.1. Study Characteristics

Thirty-three studies reported 41 treatment arms in 1238 participants (mean age 59 years, $55 \%$ males) $[34,35,38,40,43,44,47,49,50,52,56,58-60,62-66,68,70-72,74,76,78,80-82,84$, $87,90,93]$. Simvastatin was used in 17 arms $[47,52,56,58-60,62,66,78,81,82,90,93]$, atorvastatin in $9[43,44,63,70,72,74,80,87]$, pravastatin in $7[34,38,40,65,84]$, fluvastatin in $3[35,49,50]$, pitavastatin in $3[68,76]$, and rosuvastatin [71] and combination of various statins [64] in 1, respectively. Treatment duration ranged between 2 and 24 weeks (Table 1).

3.2.2. Risk of Bias

The risk of bias was low in all studies $[34,35,38,40,43,44,47,49,50,52,56,58-60,62-66,68$, 70-72,74,76,78,80-82,84,87,90,93] (Table 2). 
Table 2. The Joanna Briggs Institute critical appraisal checklist.

\begin{tabular}{|c|c|c|c|c|c|c|c|c|c|}
\hline Study & $\begin{array}{l}\text { Were the Criteria } \\
\text { for Inclusion in } \\
\text { the Sample } \\
\text { Clearly Defined? }\end{array}$ & $\begin{array}{l}\text { Were the Study } \\
\text { Subjects and the } \\
\text { Setting } \\
\text { Described in } \\
\text { Detail? }\end{array}$ & $\begin{array}{l}\text { Was the Exposure } \\
\text { Measured in a } \\
\text { Valid and } \\
\text { Reliable Way? }\end{array}$ & $\begin{array}{c}\text { Were Objective, } \\
\text { Standard Criteria } \\
\text { Used for } \\
\text { Measurement of } \\
\text { the Condition? }\end{array}$ & $\begin{array}{c}\text { Were } \\
\text { Confounding } \\
\text { Factors } \\
\text { Identified? }\end{array}$ & $\begin{array}{l}\text { Were Strategies } \\
\text { to Deal with } \\
\text { Confounding } \\
\text { Factors Stated? }\end{array}$ & $\begin{array}{l}\text { Were the } \\
\text { Outcomes } \\
\text { Measured in a } \\
\text { Valid and } \\
\text { Reliable Way? }\end{array}$ & $\begin{array}{c}\text { Was Appropriate } \\
\text { Statistical } \\
\text { Analysis Used? }\end{array}$ & Risk of Bias \\
\hline Koh KK [33] & Yes & Yes & Yes & Yes & Yes & Yes & Yes & Yes & Low \\
\hline Rauch U [34] & Yes & Yes & Yes & Yes & No & No & Yes & No & Low \\
\hline Romano M [35] & Yes & Yes & Yes & Yes & No & No & Yes & No & Low \\
\hline Sbarouni E [36] & Yes & Yes & Yes & Yes & Yes & Yes & Yes & Yes & Low \\
\hline Alonso R [37] & Yes & Yes & Yes & Yes & No & No & Yes & No & Low \\
\hline Blann AD [38] & Yes & Yes & Yes & Yes & No & No & Yes & No & Low \\
\hline Sardo MA [39] & Yes & Yes & Yes & Yes & No & No & Yes & Yes & Low \\
\hline Solheim S [40] & Yes & Yes & Yes & Yes & Yes & Yes & Yes & Yes & Low \\
\hline van Haelst PL [41] & Yes & Yes & Yes & Yes & No & No & Yes & No & Low \\
\hline Atalar E [42] & Yes & Yes & Yes & Yes & No & No & Yes & No & Low \\
\hline Seljeflot I [43] & Yes & Yes & Yes & Yes & No & No & Yes & Yes & Low \\
\hline Dalla Nora [45] & Yes & Yes & Yes & Yes & Yes & Yes & Yes & Yes & Low \\
\hline Empen K [46] & Yes & Yes & Yes & Yes & Yes & Yes & Yes & Yes & Low \\
\hline Ferroni P [47] & Yes & Yes & Yes & Yes & No & No & Yes & No & Low \\
\hline Hernández C [48] & Yes & Yes & Yes & Yes & No & No & Yes & No & Low \\
\hline Homma Y [49] & Yes & Yes & Yes & Yes & No & No & Yes & No & Low \\
\hline Malyszko J [50] & Yes & Yes & Yes & Yes & No & No & Yes & No & Low \\
\hline Nawawi H [51] & Yes & Yes & Yes & Yes & No & No & Yes & No & Low \\
\hline Puccetti L [52] & Yes & Yes & Yes & Yes & No & No & Yes & No & Low \\
\hline van Haelst PL [53] & Yes & Yes & Yes & Yes & No & No & Yes & No & Low \\
\hline Brown SL [54] & Yes & Yes & Yes & Yes & No & No & Yes & No & Low \\
\hline Ceriello A [55] & Yes & Yes & Yes & Yes & Yes & Yes & Yes & Yes & Low \\
\hline Cha JK [56] & Yes & Yes & Yes & Yes & No & No & Yes & No & Low \\
\hline Koh KK [57] & Yes & Yes & Yes & Yes & No & No & Yes & Yes & Low \\
\hline Malyszko J [58] & Yes & Yes & Yes & Yes & $\mathrm{No}$ & No & Yes & $\mathrm{No}$ & Low \\
\hline
\end{tabular}


Table 2. Cont.

\begin{tabular}{|c|c|c|c|c|c|c|c|c|c|}
\hline Study & $\begin{array}{l}\text { Were the Criteria } \\
\text { for Inclusion in } \\
\text { the Sample } \\
\text { Clearly Defined? }\end{array}$ & $\begin{array}{l}\text { Were the Study } \\
\text { Subjects and the } \\
\text { Setting } \\
\text { Described in } \\
\text { Detail? }\end{array}$ & $\begin{array}{l}\text { Was the Exposure } \\
\text { Measured in a } \\
\text { Valid and } \\
\text { Reliable Way? }\end{array}$ & $\begin{array}{c}\text { Were Objective, } \\
\text { Standard Criteria } \\
\text { Used for } \\
\text { Measurement of } \\
\text { the Condition? }\end{array}$ & $\begin{array}{c}\text { Were } \\
\text { Confounding } \\
\text { Factors } \\
\text { Identified? }\end{array}$ & $\begin{array}{l}\text { Were Strategies } \\
\text { to Deal with } \\
\text { Confounding } \\
\text { Factors Stated? }\end{array}$ & $\begin{array}{l}\text { Were the } \\
\text { Outcomes } \\
\text { Measured in a } \\
\text { Valid and } \\
\text { Reliable Way? }\end{array}$ & $\begin{array}{l}\text { Was Appropriate } \\
\text { Statistical } \\
\text { Analysis Used? }\end{array}$ & Risk of Bias \\
\hline Nomura S [59] & Yes & Yes & Yes & Yes & No & No & Yes & No & Low \\
\hline Skrha J [60] & Yes & Yes & Yes & Yes & No & No & Yes & No & Low \\
\hline Akçay MN [61] & Yes & Yes & Yes & Yes & No & No & Yes & No & Low \\
\hline Undas A [62] & Yes & Yes & Yes & Yes & No & No & Yes & No & Low \\
\hline Bleske BE [63] & Yes & Yes & Yes & Yes & Yes & Yes & Yes & Yes & Low \\
\hline Marschang P [64] & Yes & Yes & Yes & Yes & No & No & Yes & No & Low \\
\hline Peverill RE [65] & Yes & Yes & Yes & Yes & No & No & Yes & No & Low \\
\hline Potaczek PD [66] & Yes & Yes & Yes & Yes & No & No & Yes & No & Low \\
\hline Alber HF [67] & Yes & Yes & Yes & Yes & No & No & Yes & No & Low \\
\hline Inami N [68] & Yes & Yes & Yes & Yes & No & No & Yes & Yes & Low \\
\hline Jeff LS [69] & Yes & Yes & Yes & Yes & No & No & Yes & No & Low \\
\hline Barreto AC [71] & Yes & Yes & Yes & Yes & No & No & Yes & Yes & Low \\
\hline Bolewski A [72] & Yes & Yes & Yes & Yes & No & No & Yes & No & Low \\
\hline Del Papa N [73] & Yes & Yes & Yes & Yes & No & No & Yes & No & Low \\
\hline Forst T [74] & Yes & Yes & Yes & Yes & No & No & Yes & No & Low \\
\hline Hogue JC [75] & Yes & Yes & Yes & Yes & No & No & Yes & No & Low \\
\hline Nomura S [76] & Yes & Yes & Yes & Yes & Yes & Yes & Yes & Yes & Low \\
\hline Stulc T [77] & Yes & Yes & Yes & Yes & No & No & Yes & No & Low \\
\hline Wang L [78] & Yes & Yes & Yes & Yes & No & No & Yes & No & Low \\
\hline Baldassarre D [79] & Yes & Yes & Yes & Yes & No & No & Yes & No & Low \\
\hline Grip O [80] & Yes & Yes & Yes & Yes & No & No & Yes & No & Low \\
\hline Nomura S [81] & Yes & Yes & Yes & Yes & Yes & Yes & Yes & Yes & Low \\
\hline Prázný M [82] & Yes & Yes & Yes & Yes & No & No & Yes & No & Low \\
\hline Serrano CV [83] & Yes & Yes & Yes & Yes & No & No & Yes & No & Low \\
\hline Fichtenbaum CJ [84] & Yes & Yes & Yes & Yes & No & No & Yes & No & Low \\
\hline
\end{tabular}


Table 2. Cont

\begin{tabular}{|c|c|c|c|c|c|c|c|c|c|}
\hline Study & $\begin{array}{l}\text { Were the Criteria } \\
\text { for Inclusion in } \\
\text { the Sample } \\
\text { Clearly Defined? }\end{array}$ & $\begin{array}{l}\text { Were the Study } \\
\text { Subjects and the } \\
\text { Setting } \\
\text { Described in } \\
\text { Detail? }\end{array}$ & $\begin{array}{l}\text { Was the Exposure } \\
\text { Measured in a } \\
\text { Valid and } \\
\text { Reliable Way? }\end{array}$ & $\begin{array}{c}\text { Were Objective, } \\
\text { Standard Criteria } \\
\text { Used for } \\
\text { Measurement of } \\
\text { the Condition? }\end{array}$ & $\begin{array}{c}\text { Were } \\
\text { Confounding } \\
\text { Factors } \\
\text { Identified? }\end{array}$ & $\begin{array}{l}\text { Were Strategies } \\
\text { to Deal with } \\
\text { Confounding } \\
\text { Factors Stated? }\end{array}$ & $\begin{array}{l}\text { Were the } \\
\text { Outcomes } \\
\text { Measured in a } \\
\text { Valid and } \\
\text { Reliable Way? }\end{array}$ & $\begin{array}{l}\text { Was Appropriate } \\
\text { Statistical } \\
\text { Analysis Used? }\end{array}$ & Risk of Bias \\
\hline Kater AL [85] & Yes & Yes & Yes & Yes & No & No & Yes & Yes & Low \\
\hline Kirmizis K [86] & Yes & Yes & Yes & Yes & No & No & Yes & Yes & Low \\
\hline Xu DY [87] & Yes & Yes & Yes & Yes & No & No & Yes & No & Low \\
\hline Wu YW [88] & Yes & Yes & Yes & Yes & No & No & Yes & No & Low \\
\hline Altun I [89] & Yes & Yes & Yes & Yes & No & No & Yes & No & Low \\
\hline Pawelczyk M [90] & Yes & Yes & Yes & Yes & No & No & Yes & No & Low \\
\hline Hernandez-Mijares A [91] & Yes & Yes & Yes & Yes & No & No & Yes & No & Low \\
\hline Barale C [92] & Yes & Yes & Yes & Yes & No & No & Yes & No & Low \\
\hline Kotyla PJ [93] & Yes & Yes & Yes & Yes & $\mathrm{No}$ & No & Yes & No & Low \\
\hline
\end{tabular}




\subsubsection{Results of Individual Studies and Syntheses}

The forest plot for circulating P-Selectin concentrations before and after statin treatment is shown in Figure 2. In six arms $[34,44,60,68,74,78]$, concentrations were higher after treatment (mean difference range, 0.10 to 0.62 ); however, the difference was statistically significant only in one [34]. In the remaining arms [35,38,40,43,47,49,50,52,56,58,59,62$66,70-72,76,80-82,84,87,90,93]$, P-Selectin concentrations were lower after treatment (mean difference range, -0.03 to -4.11 ) with a significant difference reported in nine $[47,49,52$, $56,59,64,65,87,90]$. The large between-study heterogeneity observed $\left(\mathrm{I}^{2}=74.1 \%, p<0.001\right)$ prompted the use of random-effects models. Pooled results showed that P-Selectin concentrations were significantly lower after statin treatment $(\mathrm{SMD}=-0.39,95 \% \mathrm{CI}-0.55$ to $-0.22, p<0.001)$.

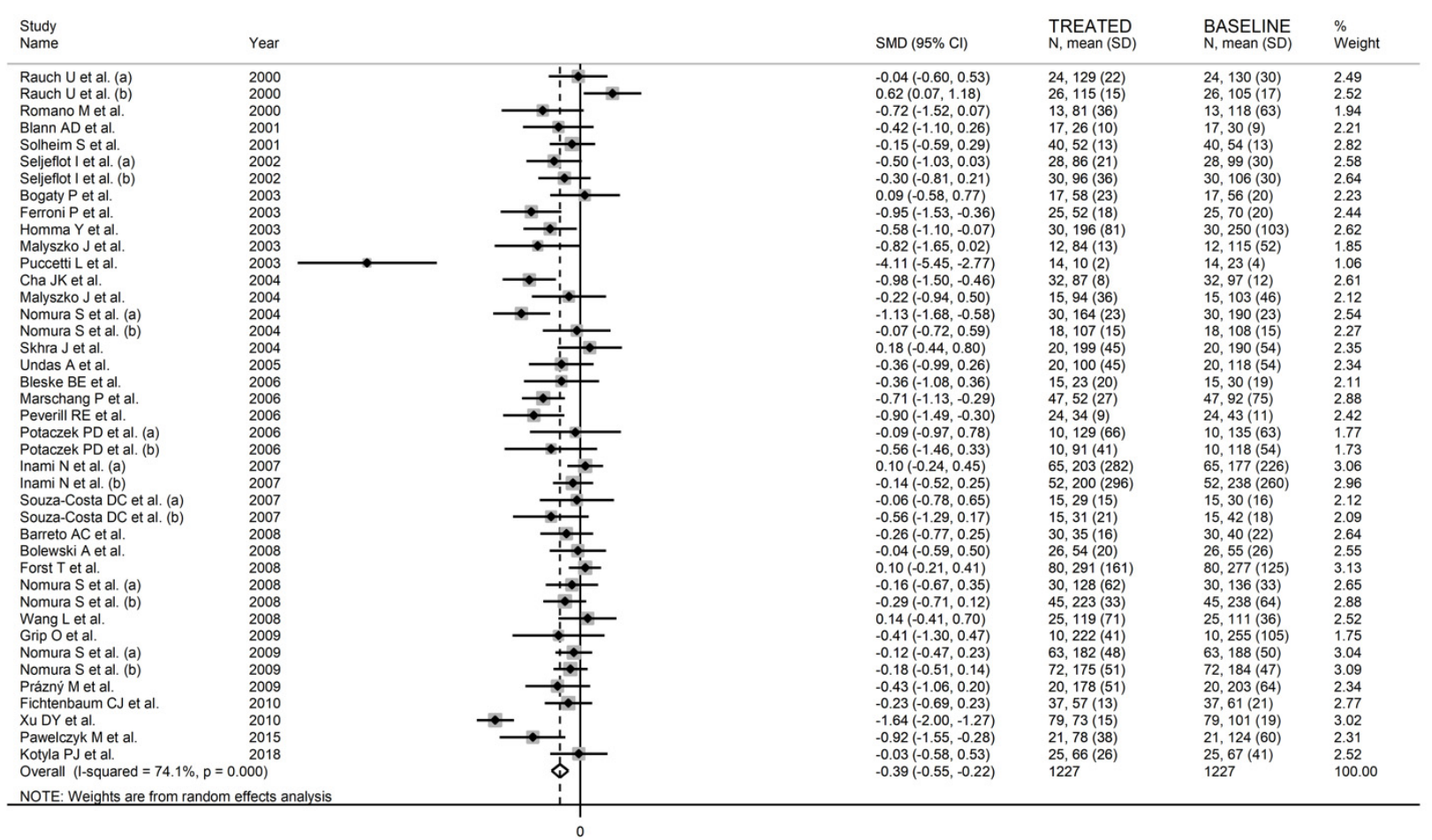

Figure 2. Forest plot of circulating P-Selectin concentrations before and after statin treatment.

In sensitivity analysis, the corresponding pooled SMD values were not substantially altered when individual studies were sequentially removed (effect size range, between -0.41 and -0.33 , Figure 3A). However, the funnel plot analysis, reported in Figure 3B, detected a distortive effect of one study [52]. Removing this study mildly attenuated both the effect size (SMD $=-0.35,95 \% \mathrm{CI}-0.50$ to $-0.20, p<0.001$ ) and the heterogeneity $\left(\mathrm{I}^{2}=68.5 \%, p<0.001\right)$.

\subsubsection{Publication Bias}

The analysis of the remaining 40 arms, after removing the previously described study [52], did not show significant publication bias (Begg's test, $p=0.12$; Egger's test, $p=0.57$ ). The "trim-and-fill" method identified one potential missing study to be added to the left side of the funnel plot to ensure symmetry (adjusted SMD $=-0.37,95 \% \mathrm{CI}-0.52$ to $-0.22, p<0.001$; Figure 4 ). 


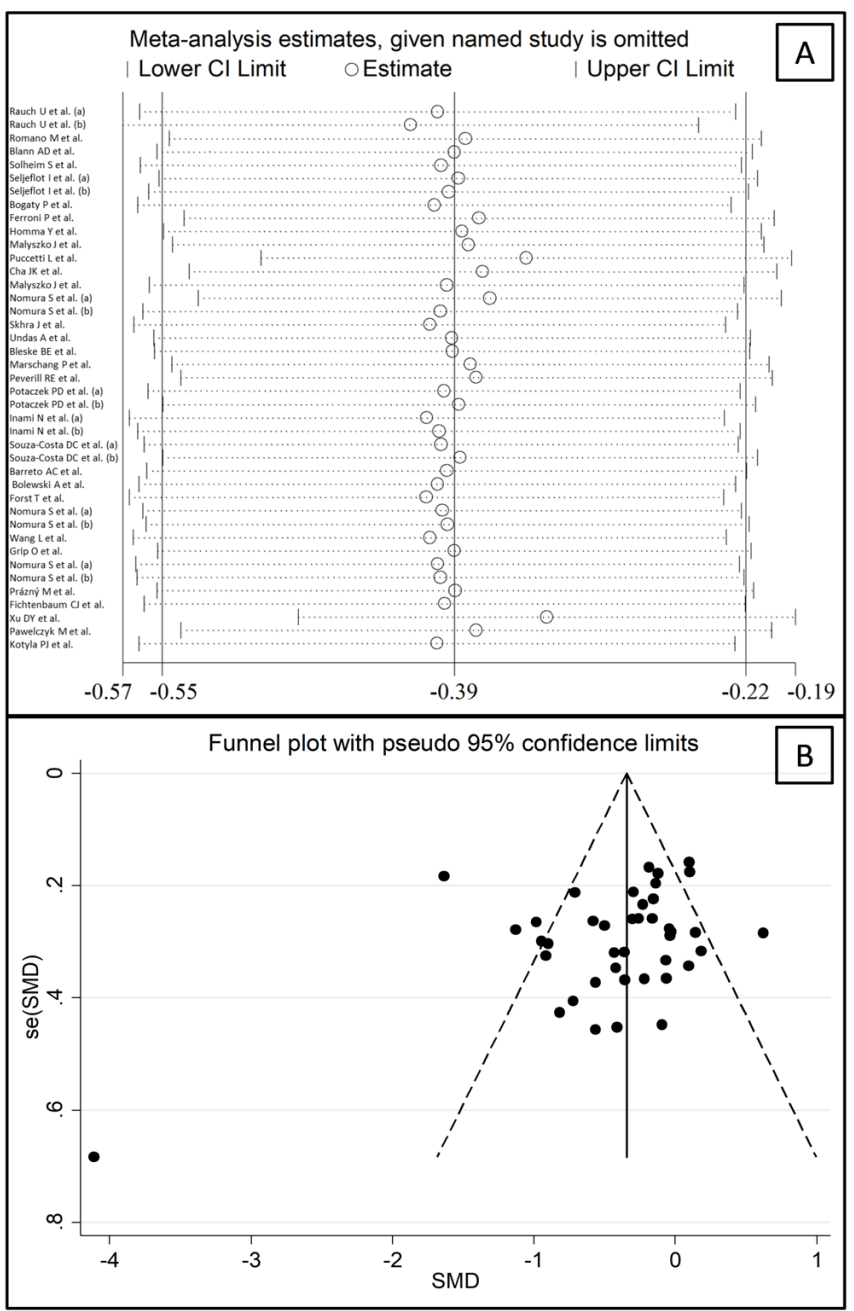

Figure 3. (A) Sensitivity analysis of the association between P-Selectin and statin treatment. The influence of individual studies on the overall standardized mean difference (SMD) is shown. The middle vertical axis indicates the overall SMD, and the two vertical axes indicate the $95 \%$ confidence intervals (CIs). The hollow circles represent the pooled SMD when the remaining study is omitted from the meta-analysis. The two ends of each broken line represent the 95\% CIs. (B) Funnel plot of studies investigating P-Selectin concentrations before and after statin treatment.

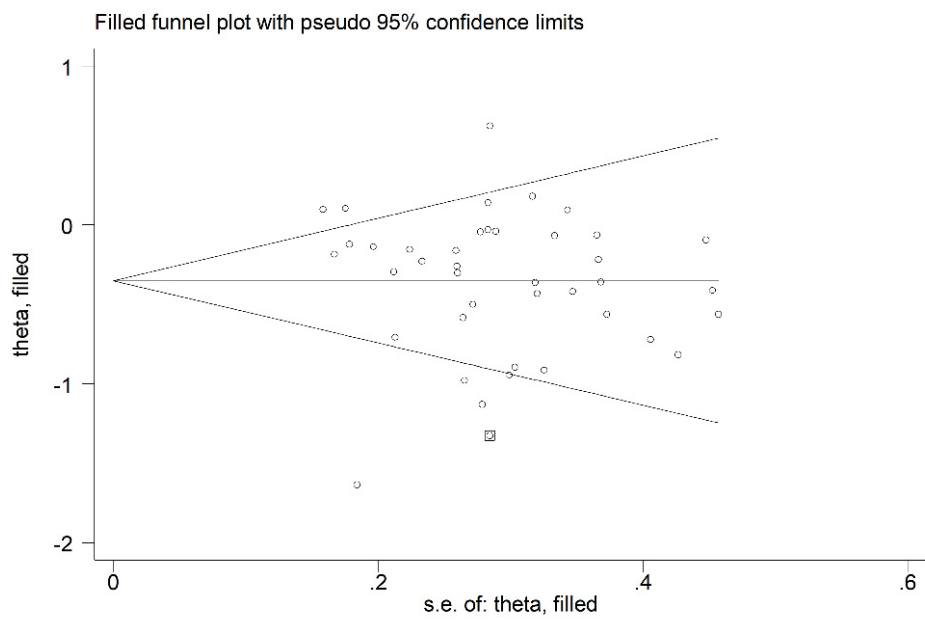

Figure 4. Funnel plot of P-Selectin concentrations before and after statin treatment after "trimmingand-filling". Dummy studies and genuine studies are represented by enclosed circles and free circles, respectively. 


\subsubsection{Meta-Regression and Subgroup Analysis}

In univariate meta-regression, there were no significant associations between effect size and age $(t=-0.61, p=0.55)$; proportion of males $(t=0.03, p=0.98)$; body mass index $(\mathrm{t}=0.43, p=0.67)$; publication year $(\mathrm{t}=-0.37, p=0.71)$; sample size $(\mathrm{t}=-0.23$, $p=0.81)$; baseline total cholesterol $(\mathrm{t}=0.80, p=0.43)$, LDL-cholesterol $(\mathrm{t}=0.95, p=0.35)$, HDL-cholesterol $(\mathrm{t}=0.36, p=0.72)$, and triglycerides $(\mathrm{t}=0.79, p=0.44)$; and treatment duration $(t=0.19, p=0.85)$. In sub-group analysis, the P-Selectin-lowering effect with lipophilic statins (SMD $=-0.37,95 \% \mathrm{CI}-0.55$ to $-0.18, p<0.001 ; \mathrm{I}^{2}=72.2 \%, p<0.001$ ) was relatively larger than that with hydrophilic statins (SMD $=-0.19,95 \% \mathrm{CI}-0.38$ to -0.004 , $p=0.046 ; \mathrm{I}^{2}=22.7 \%, p=0.25$, Figure 5); however, this difference was not statistically significant $(p=0.45)$.

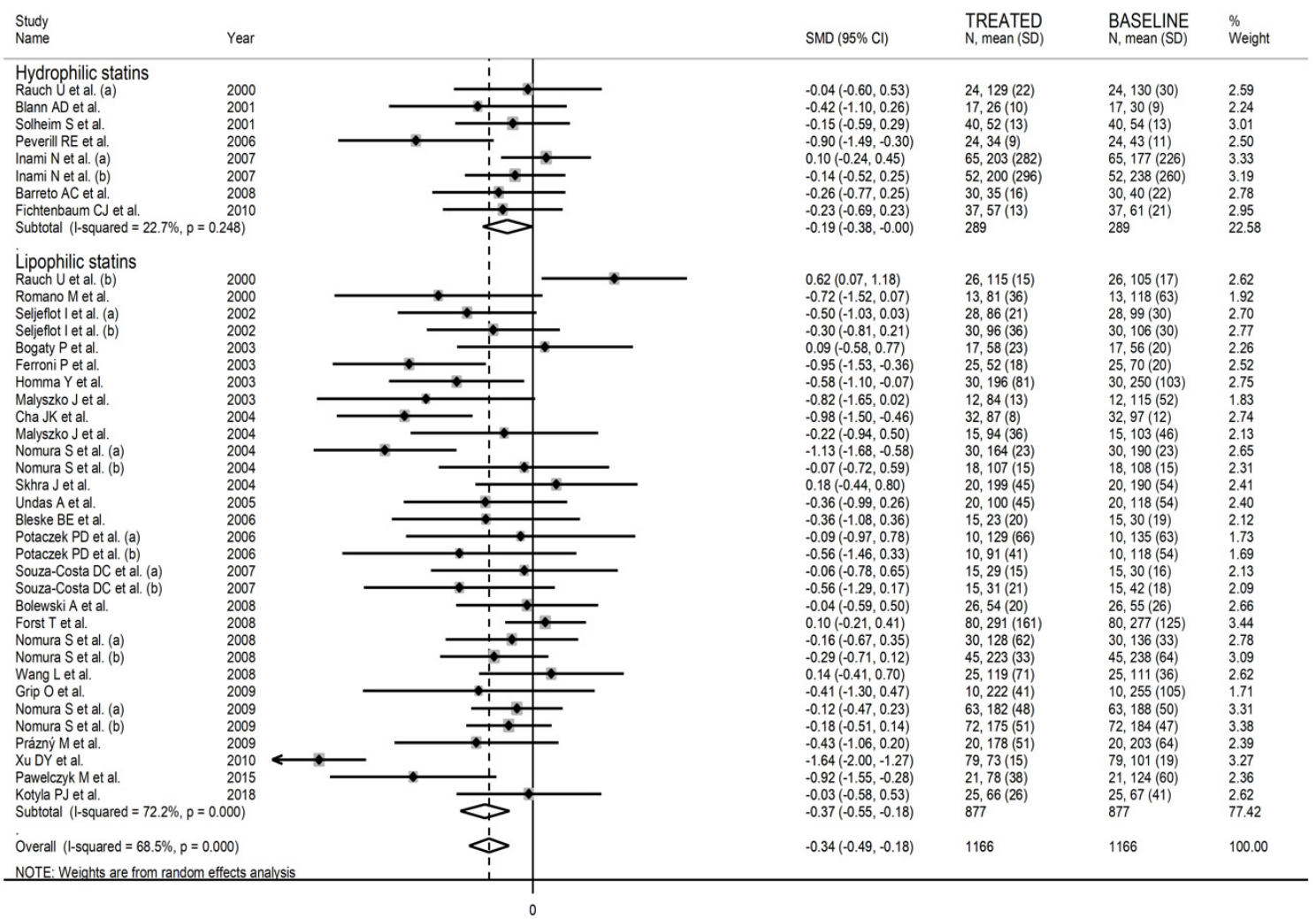

Figure 5. Forest plot of P-Selectin concentrations before and after statin treatment according to statin class (hydrophilic or lipophilic).

When considering individual agents, a significant lowering effect was observed with simvastatin (SMD $=-0.32,95 \% \mathrm{CI}-0.57$ to $-0.08, p=0.005 ; \mathrm{I}^{2}=64.5 \%, p<0.001$ ) and fluvastatin (SMD $=-0.67,95 \% \mathrm{CI}-1.05$ to $\left.-0.28, p=0.001 ; \mathrm{I}^{2}=0.0 \%, p=0.89\right)$, but not with atorvastatin $\left(\mathrm{SMD}=-0.39,95 \% \mathrm{CI}-0.88\right.$ to $\left.0.11, p=0.12 ; \mathrm{I}^{2}=86.5 \%, p<0.001\right)$, pravastatin (SMD $=-0.19,95 \% \mathrm{CI}-0.41$ to $\left.0.02, p=0.06 ; \mathrm{I}^{2}=32.9 \%, p=0.18\right)$, or pitavastatin $\left(\mathrm{SMD}=-0.21,95 \% \mathrm{CI}-0.44\right.$ to $0.02, p=0.07 ; \mathrm{I}^{2}=0.0 \%, p=0.98$ ) (Figure 6 ). As reported in Figure 7, a significant P-Selectin-lowering effect was reported in studies conducted in Asia (SMD $=-0.47,95 \% \mathrm{CI}-0.82$ to $-0.12, p=0.008 ; \mathrm{I}^{2}=86.3 \%, p<0.001$ ) and Europe $\left(\mathrm{SMD}=-0.35,95 \% \mathrm{CI}-0.51\right.$ to $\left.-0.18, p<0.001 ; \mathrm{I}^{2}=32.9 \%, p=0.08\right)$ but not America $\left(\mathrm{SMD}=-0.05,95 \% \mathrm{CI}-0.28\right.$ to $\left.0.17, p=0.63 ; \mathrm{I}^{2}=20.7 \%, p=0.26\right)$. 


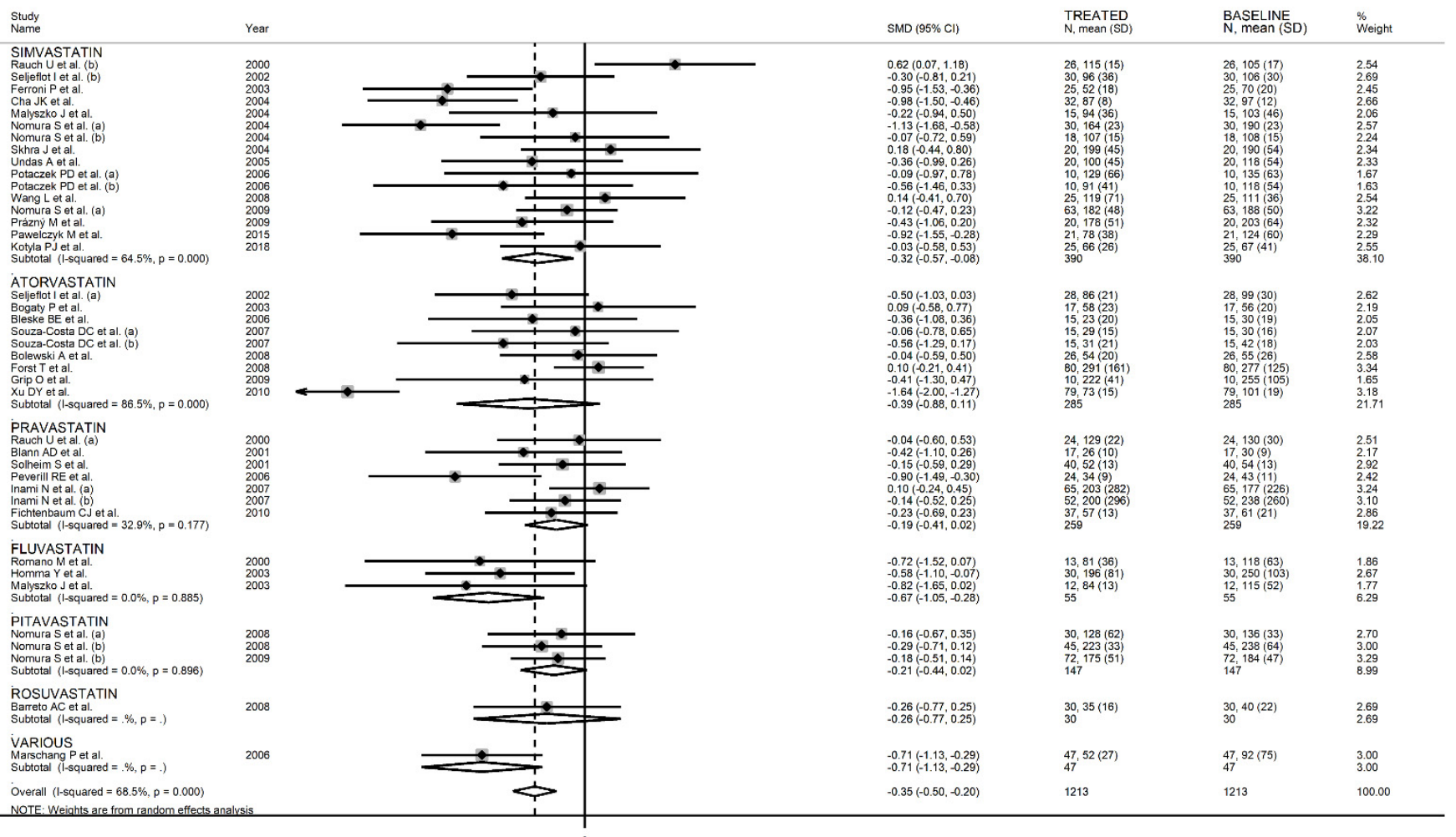

Figure 6. Forest plot of P-Selectin concentrations before and after statin treatment according to individual agents.

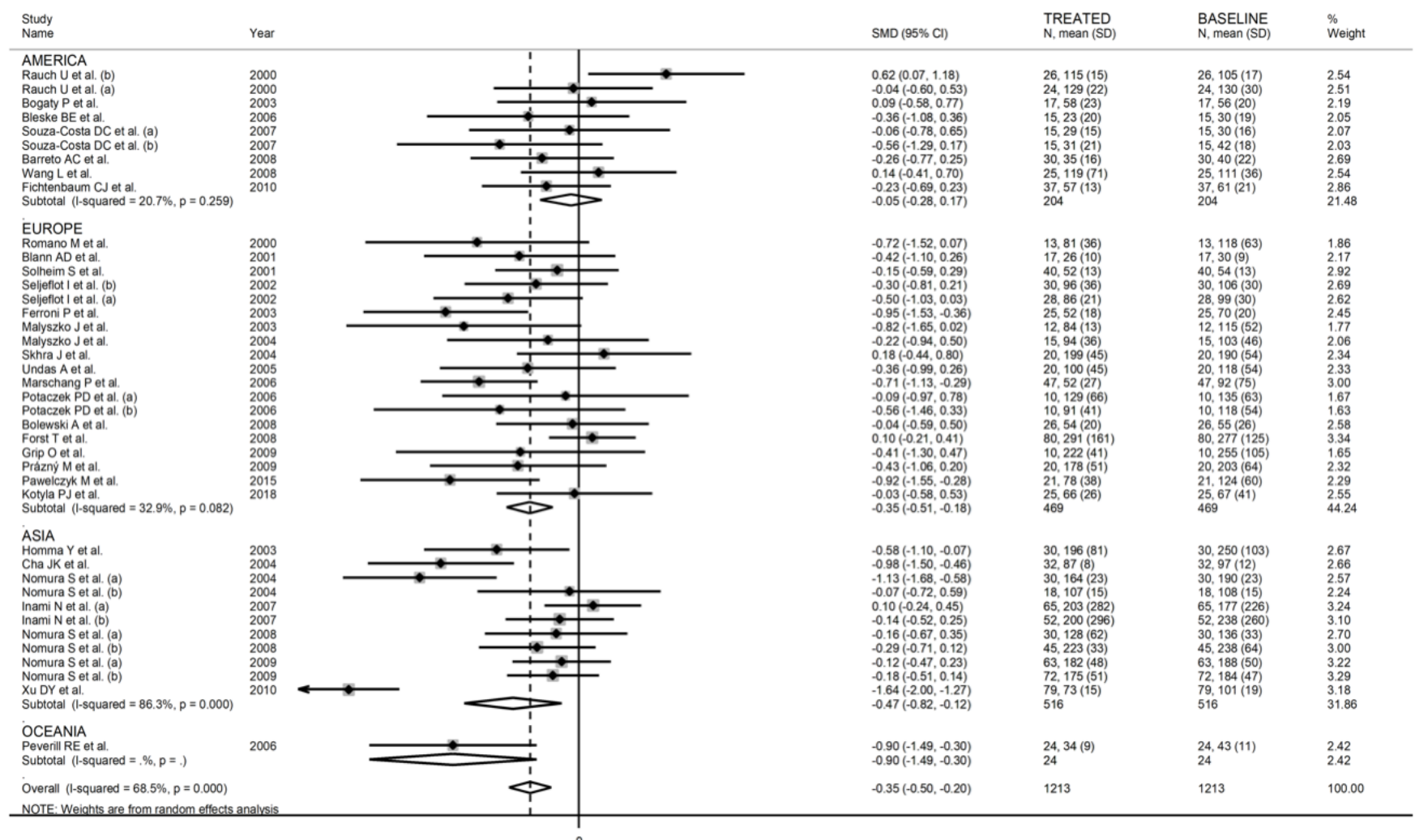

Figure 7. Forest plot of P-Selectin concentrations before and after statin treatment according to continent where the study was conducted. 
We further sought to identify more homogeneous study sub-groups according to statin used and continent. In a sub-group of nine studies (10 treatment arms) conducted in Europe using atorvastatin $[43,47,58,60,62,66,82,90,93]$, the significant reduction in P-Selectin concentrations ( $\mathrm{SMD}=-0.37,95 \% \mathrm{CI}-0.60$ to $-0.14, p=0.002$ ) was associated with a markedly lower heterogeneity $\left(\mathrm{I}^{2}=24.1 \%, p=0.22\right)$ (Figure 8 ).

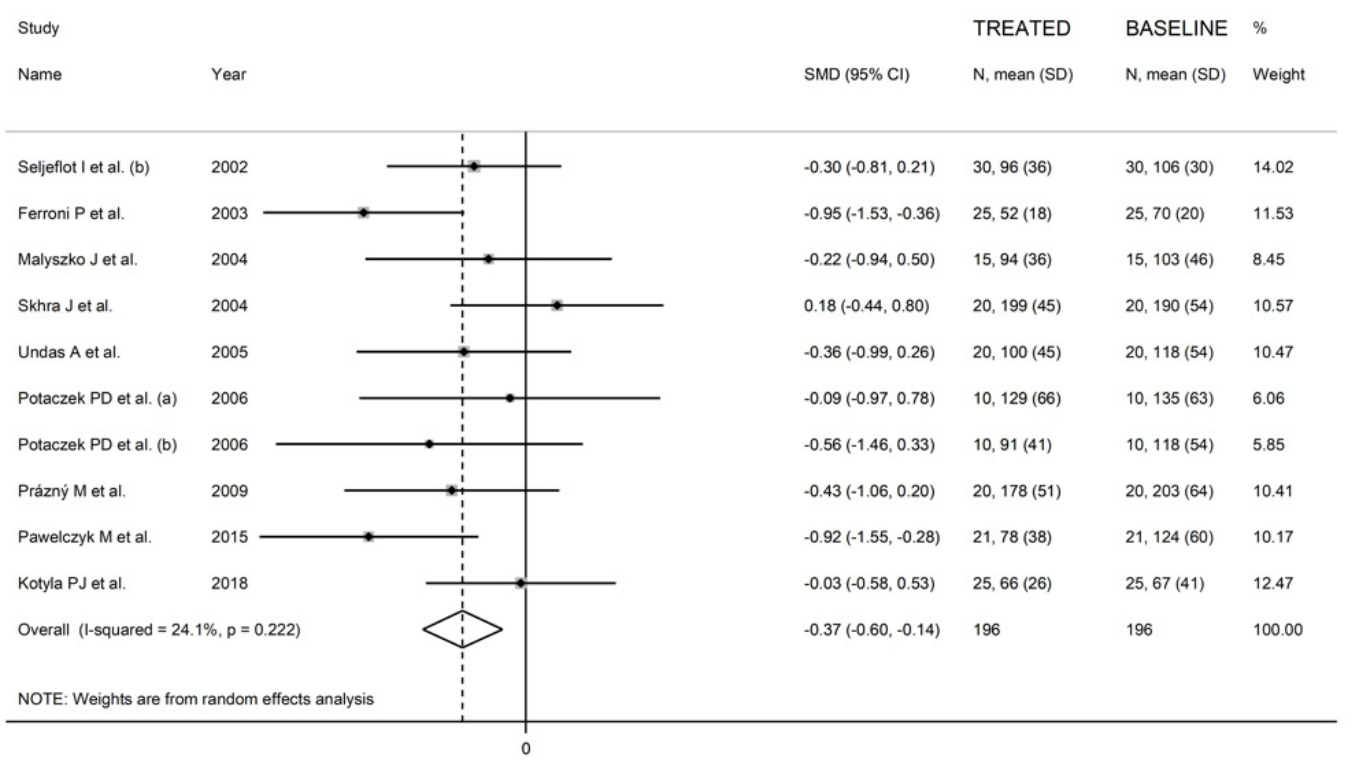

Figure 8. Forest plot of a sub-group of 10 studies examining P-Selectin concentrations, homogeneous for statin used and continent.

\subsubsection{Certainty of Evidence}

The initial level of certainty for P-Selectin SMD values was considered moderate because of the interventional nature of the studies (rating $3, \oplus \oplus \oplus \ominus$ ). After considering the low risk of bias in all studies (upgrade one level), a generally large heterogeneity was partially explained by the specific statin used and the continent where the study was conducted (no rating change required), the lack of indirectness (no rating change required), the relatively low imprecision (relatively narrow confidence intervals without threshold crossing, no rating change required), the relatively small effect size ( $\mathrm{SMD}=-0.39$, downgrade one level), and the absence of publication bias (no rating change required); the overall level of certainty remained moderate (rating $3, \oplus \oplus \oplus \ominus$ ).

\subsection{Meta-Analysis of Soluble L-Selectin}

\subsubsection{Study Characteristics}

Four studies reported six treatment arms in 186 participants (mean age 55 years, $59 \%$ males) $[34,42,76,93]$. The statin used was simvastatin in two arms [34,93], pitavastatin in two [76], and atorvastatin [42] and pravastatin [34] in one, respectively. Duration of therapy ranged between 4 and 24 weeks (Table 1).

\subsubsection{Risk of Bias}

The risk of bias was low in all studies $[34,42,76,93]$ (Table 2).

\subsubsection{Results of Individual Studies and Syntheses}

The forest plot for circulating L-Selectin concentrations before and after statin treatment is shown in Figure 9. In five arms [34,42,76], concentrations were lower after treatment (mean difference range, -1.50 to -0.13 ), and the difference was statistically significant in two [34,76]. In the remaining arm [93], L-Selectin concentrations were non-significantly higher after treatment. Random-effects models were used in view of the large heterogeneity observed $\left(\mathrm{I}^{2}=71.1 \%, p=0.004\right)$. Pooled results showed that L-Selectin concentrations were 
significantly lower after treatment $(\mathrm{SMD}=-0.49,95 \% \mathrm{CI}-0.89$ to $-0.10, p=0.014)$. In sensitivity analysis, the corresponding pooled SMD values were not substantially altered when individual studies were sequentially removed (effect size range, between -0.61 and -0.33 , Figure 10).

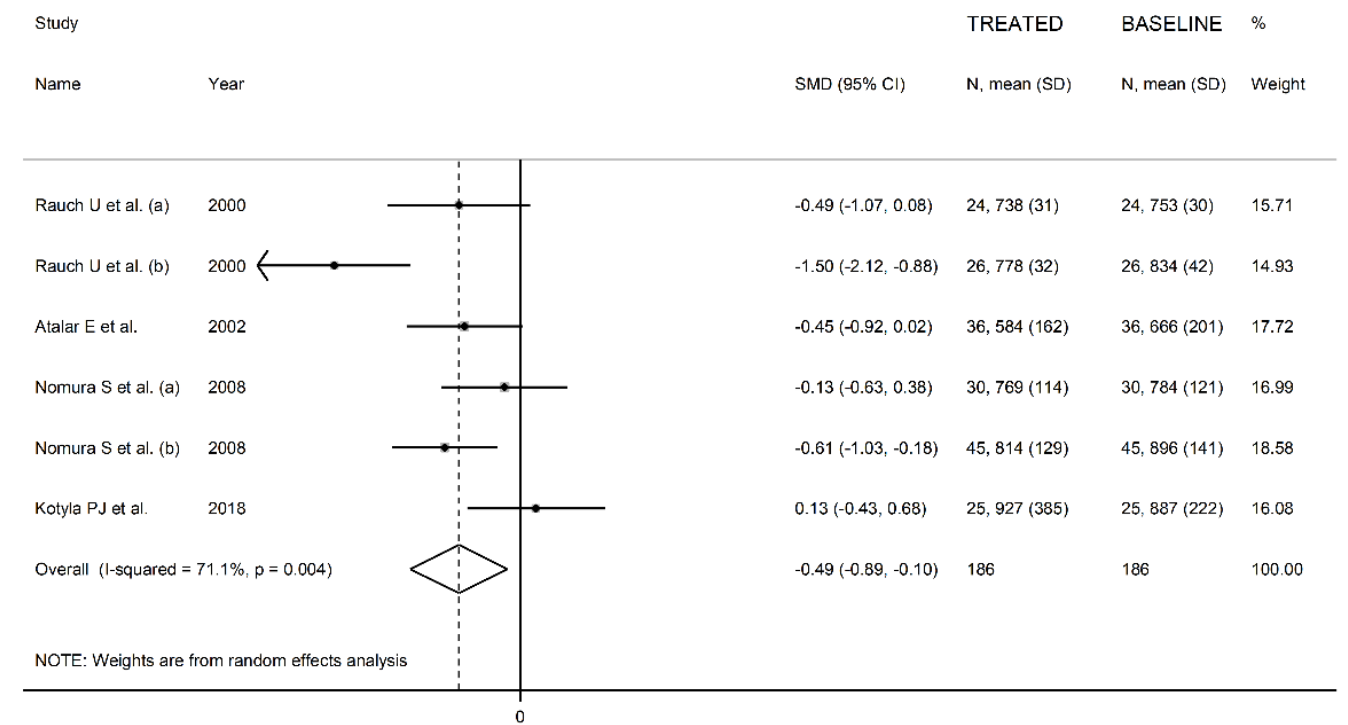

Figure 9. Forest plot of L-Selectin concentrations before and after statin treatment.

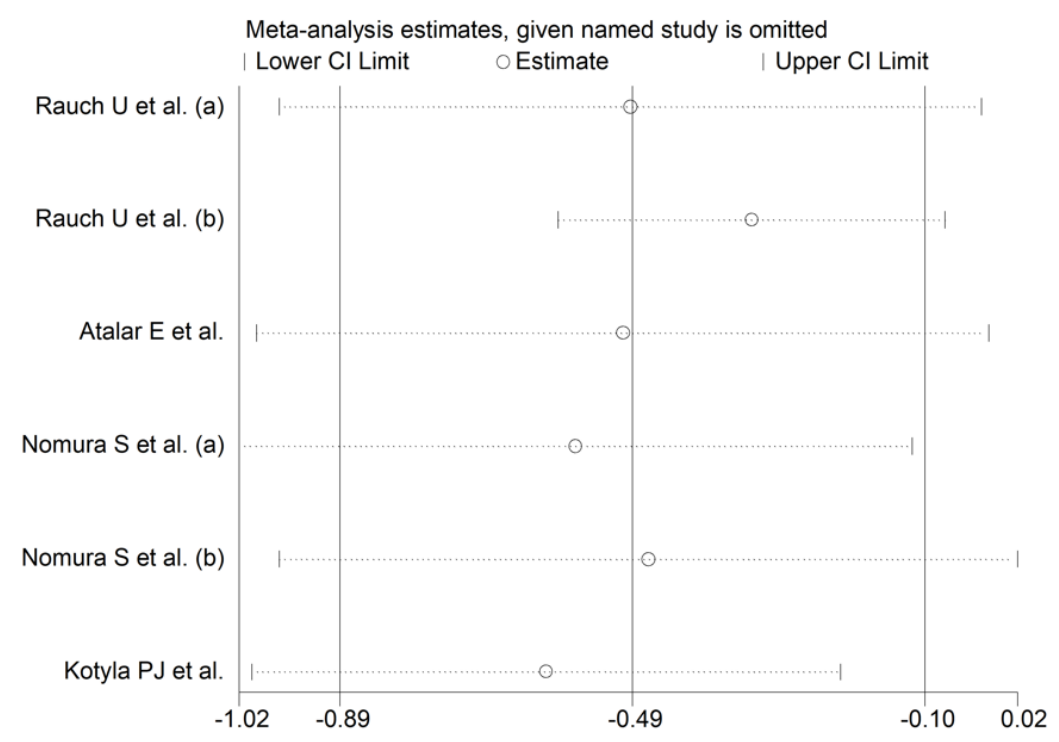

Figure 10. Sensitivity analysis of the association between L-Selectin and statin treatment. The influence of individual studies on the overall standardized mean difference (SMD) is shown. The middle vertical axis indicates the overall SMD, and the two vertical axes indicate the $95 \%$ confidence intervals (CIs). The hollow circles represent the pooled SMD when the remaining study is omitted from the meta-analysis. The two ends of each broken line represent the $95 \%$ CIs.

\subsubsection{Publication Bias}

Assessment of publication bias was not possible because of the small number of studies.

\subsubsection{Meta-Regression and Subgroup Analysis}

Meta-regression and sub-group analysis were not possible because of the small number of studies. 


\subsubsection{Certainty of Evidence}

The initial level of certainty for L-Selectin SMD values was considered moderate because of the interventional nature of the studies (rating $3, \oplus \oplus \oplus \ominus$ ). After considering the low risk of bias in all studies (upgrade one level), a large unexplained heterogeneity (downgrade one level), the lack of indirectness (no rating change required), the relatively low imprecision (relatively narrow confidence intervals without threshold crossing, no rating change required), the relatively small effect size ( $\mathrm{SMD}=-0.49$, downgrade one level), and the lack of assessment of publication bias (downgrade one level), the overall level of certainty was considered very low (rating $1, \oplus \ominus \ominus \ominus$ ).

\subsection{Meta-Analysis of Soluble E-Selectin \\ 3.4.1. Study Characteristics}

Thirty-eight studies reported 41 treatment arms in 1097 patients (mean age 55 years, $60 \%$ males) $[33,36,37,39-41,43-46,48,51,53-55,57,58,60,61,64,67,69,72,73,75-77,79,80,82,83$, $85,86,88,89,91-93]$. Simvastatin was used in 19 arms [33,36,37,39,43,53-55,57,58,60,61,73,82, $83,85,86,91-93]$, atorvastatin in 15 [43-46,51,67,72,75,77,79,80,88,89], pravastatin in 2 [40,69], pitavastatin in 2 [76], various combination of statins in 2 [48,64], and fluvastatin in 1 [41]. Duration of therapy ranged between 4 and 52 weeks (Table 1).

\subsubsection{Risk of Bias}

The risk of bias was low in all studies $[33,36,37,39-41,43-46,48,51,53-55,57,58,60,61,64$, $67,69,72,73,75-77,79,80,82,83,85,86,88,89,91-93]$ (Table 2).

\subsubsection{Results of Individual Studies and Syntheses}

The forest plot for circulating E-Selectin concentrations before and after statin treatment is shown in Figure 11. In nine arms $[43,49,67,70,74,79,82,87,93]$, concentrations were higher after treatment (mean difference range, 0.03 to 0.63 ); however, a significant difference was reported only in one [83]. Virtually identical pre- and post-treatment concentrations were reported in two arms [36,54]. In the remaining arms $[37,40,41,43-$ $46,48,51,53,55,58,61,67,73,75,76,79,80,82,85,86,88,89,91-93]$, E-Selectin concentrations were lower after treatment (mean difference range, -0.04 to -11.66 ), with a significant difference reported in 13 [37,41,44,45,48,51,61,76,79,82,92]. Extreme heterogeneity between studies was observed $\left(\mathrm{I}^{2}=90.4 \%, p<0.001\right)$, requiring the use of random-effects models. Pooled results showed that circulating E-Selectin concentrations were significantly lower after treatment (SMD $=-0.73,95 \% \mathrm{CI}-1.02$ to $-0.43, p<0.001$ ). In sensitivity analysis, the corresponding pooled SMD values were not substantially altered when individual studies were sequentially removed (effect size range, between -0.76 and -0.53 , Figure $12 \mathrm{~A}$ ). However, the funnel plot analysis, reported in Figure 12B, detected a distortive effect of three studies (four treatment arms) $[45,51,61]$. Their removal attenuated both the effect size (SMD $=-0.33,95 \% \mathrm{CI}-0.50$ to $-0.16, p<0.001)$ and the magnitude of the heterogeneity $\left(\mathrm{I}^{2}=71.4 \%, p<0.001\right)$. 


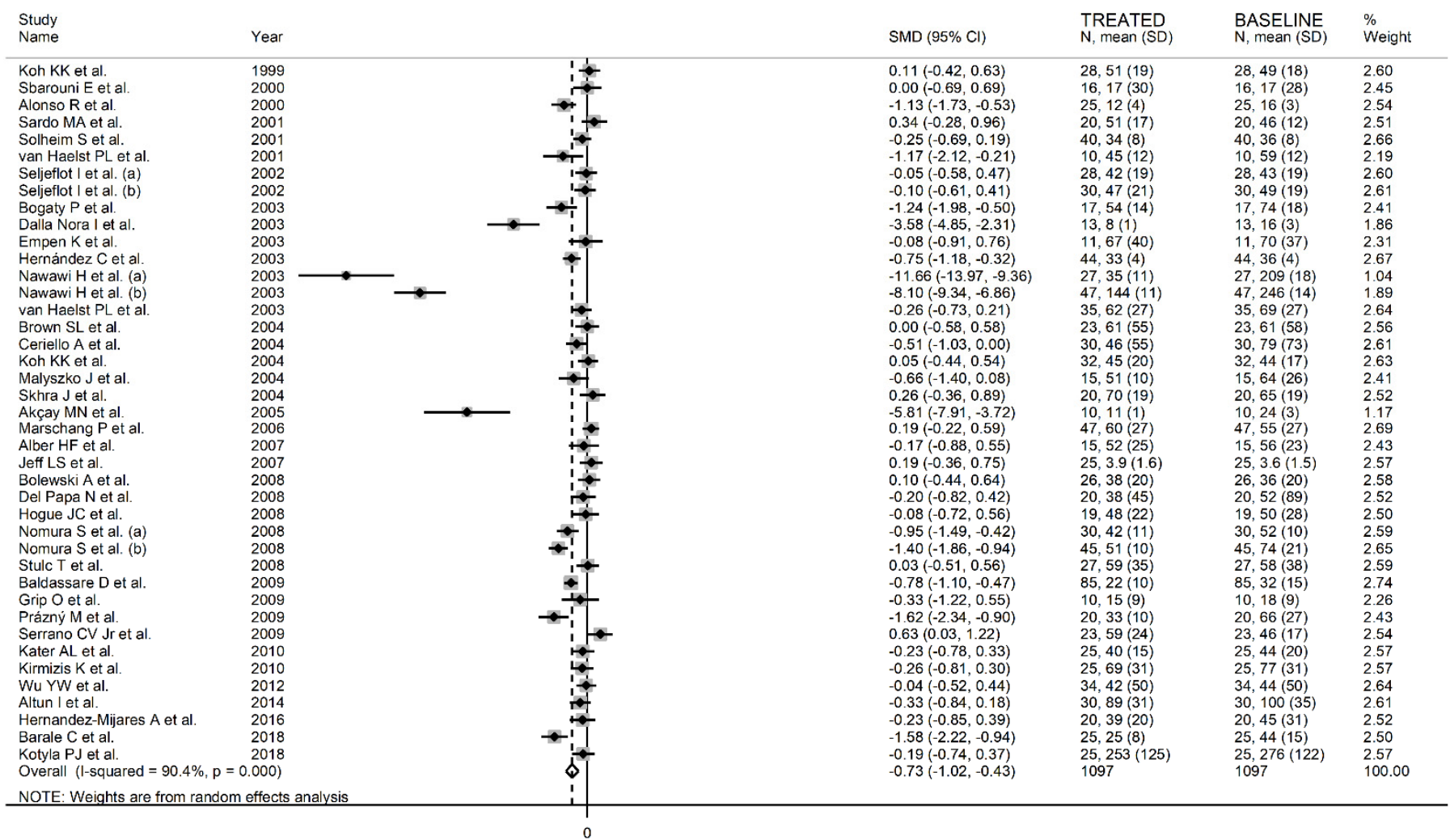

Figure 11. Forest plot of E-Selectin concentrations before and after statin treatment.

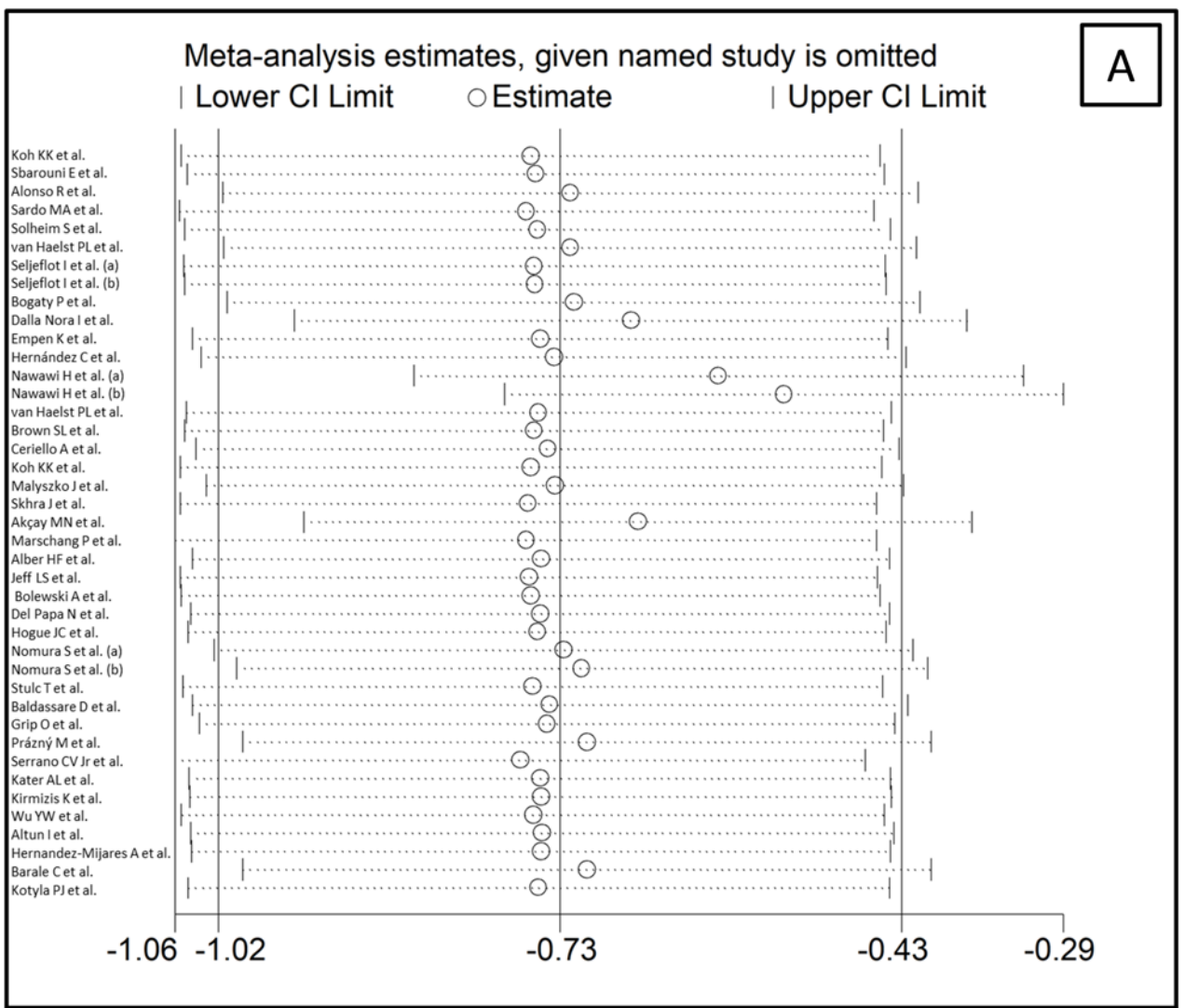

Figure 12. Cont. 


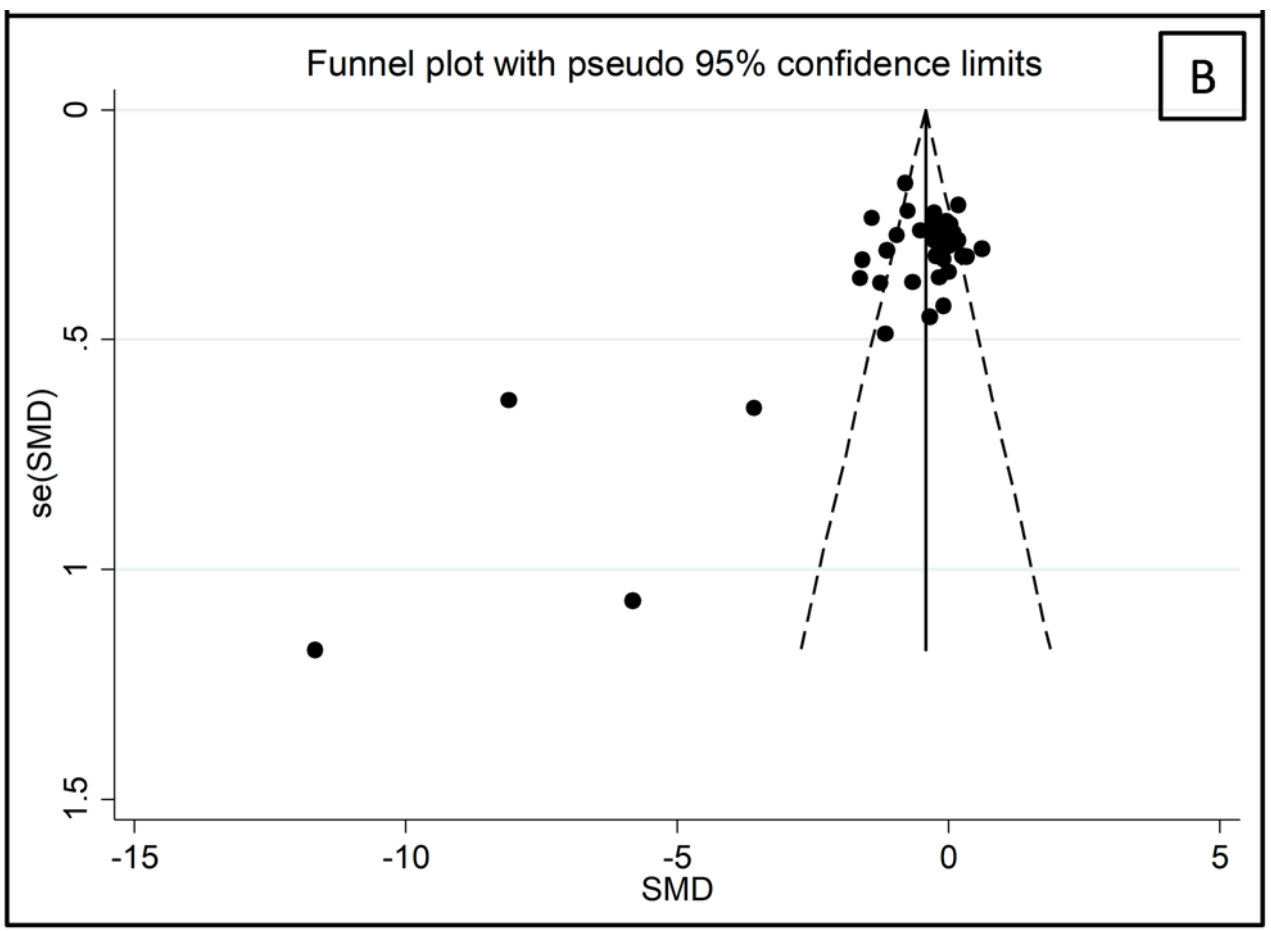

Figure 12. (A) Sensitivity analysis of the association between E-Selectin and statin treatment. The influence of individual studies on the overall standardized mean difference (SMD) is shown. The middle vertical axis indicates the overall SMD, and the two vertical axes indicate the $95 \%$ confidence intervals (CIs). The hollow circles represent the pooled SMD when the remaining study is omitted from the meta-analysis. The two ends of each broken line represent the 95\% CIs. (B) Funnel plot of studies investigating E-Selectin concentrations before and after statin treatment.

\subsubsection{Publication Bias}

The analysis of the remaining 37 treatment arms did not show publication bias (Begg's test, $p=0.57$; Egger's test, $p=0.90$ ). However, the "trim-and-fill" method identified four potential missing studies to be added to the left side of the funnel plot to ensure symmetry (adjusted SMD $=-0.41,95 \% \mathrm{CI}-0.58$ to $-0.24, p<0.001$; Figure 13 ).

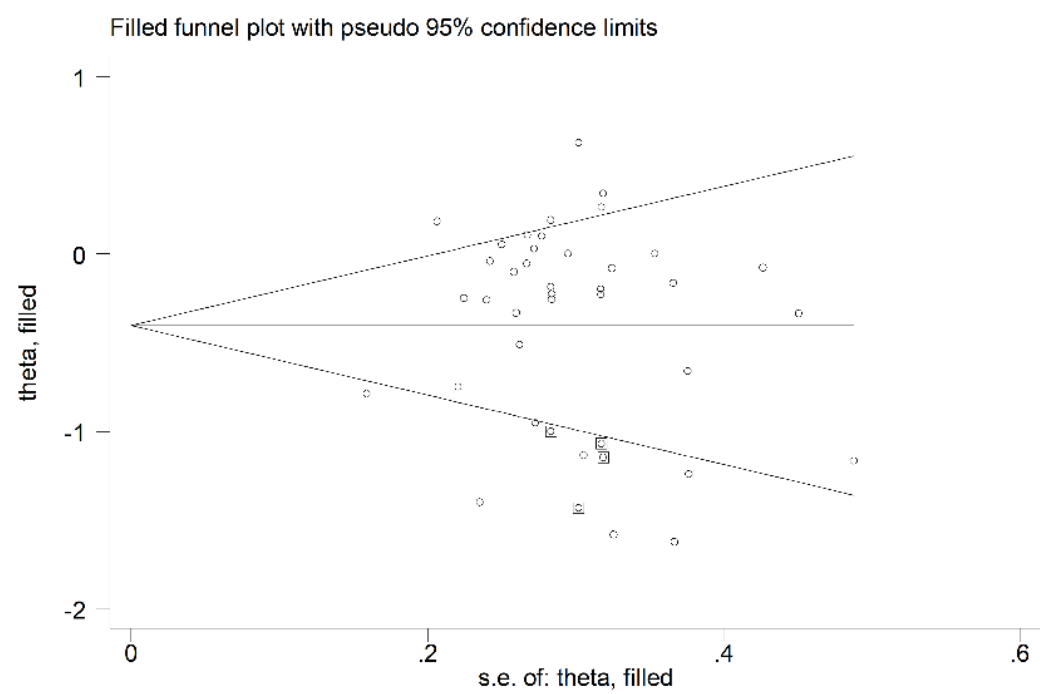

Figure 13. Funnel plot of studies investigating E-Selectin concentrations before and after statin treatment after "trimming-and-filling". Dummy studies and genuine studies are represented by enclosed circles and free circles, respectively. 


\subsubsection{Meta-Regression and Subgroup Analysis}

In univariate meta-regression, there were no significant associations between effect size and age $(t=-0.23, p=0.82)$, proportion of males $(t=-0.58, p=0.57)$, body mass index $(\mathrm{t}=0.43, p=0.67)$, publication year $(\mathrm{t}=-0.87, p=0.31)$, sample size $(\mathrm{t}=-0.90, p=0.38)$, baseline total cholesterol $(\mathrm{t}=-0.37, p=0.71)$, LDL-cholesterol $(\mathrm{t}=-0.30, p=0.77)$, HDLcholesterol $(\mathrm{t}=0.91, p=0.37)$, and triglycerides $(\mathrm{t}=0.94, p=0.36)$, and treatment duration $(\mathrm{t}=-1.44, p=0.16)$. In sub-group analysis, a significant lowering effect was observed with lipophilic (SMD $=-0.35,95 \% \mathrm{CI}-0.54$ to $-0.17, p<0.001 ; \mathrm{I}^{2}=71.4 \%, p<0.001$ ) but not hydrophilic statins (SMD $=-0.06,95 \% \mathrm{CI}-0.49$ to $0.37, p=0.65 ; \mathrm{I}^{2}=33.5 \%, p=0.22$ ) (Figure 14). When assessing individual agents, a significant lowering effect was observed with simvastatin $\left(\mathrm{SMD}=-0.28,95 \% \mathrm{CI}-0.52\right.$ to $-0.03, p=0.03 ; \mathrm{I}^{2}=70.2 \%, p<0.001$ ), atorvastatin (SMD $=-0.27,95 \% \mathrm{CI}-0.53$ to $\left.-0.02, \mathrm{p}=0.035 ; \mathrm{I}^{2}=54.1 \%, p=0.016\right)$, and pitavastatin (SMD $=-1.20,95 \% \mathrm{CI}-1.63$ to $\left.-0.76, p<0.001 ; \mathrm{I}^{2}=35.0 \%, p=0.22\right)$, but not pravastatin (SMD $=-0.06,95 \% \mathrm{CI}-0.49$ to $0.37, p=0.65 ; \mathrm{I}^{2}=33.5 \%, p=0.22$ ) (Figure 15). Moreover, as reported in Figure 16, a significant decrease in E-Selectin concentrations was reported in studies conducted in Europe (SMD $=-0.36,95 \%$ CI -0.55 to -0.16 , $p<0.001 ; \mathrm{I}^{2}=66.7 \%, p<0.001$ ) but not America (SMD $=-0.13,95 \% \mathrm{CI}-0.67$ to 0.40 $p=0.62 ; \mathrm{I}^{2}=20.7 \%, p=0.26$ ) or Asia (SMD $=-0.53,95 \% \mathrm{CI}-1.10$ to $0.03, p=0.06$; $\left.\mathrm{I}^{2}=84.7 \%, p<0.001\right)$.

\subsubsection{Certainty of Evidence}

The initial level of certainty for E-Selectin SMD values was considered moderate because of the interventional nature of the studies (rating $3, \oplus \oplus \oplus \ominus$ ). After considering the low risk of bias in all studies (upgrade one level), a large heterogeneity that was only partially attenuated after removing three studies (downgrade one level); the lack of indirectness (no rating change required); the relatively low imprecision (relatively narrow confidence intervals without threshold crossing, no rating change required); the relatively moderate effect size (SMD $=-0.73$, no rating change required); and the lack of publication bias (no rating change required), the overall level of certainty remained moderate (rating 3 , $\oplus \oplus \oplus \ominus)$.

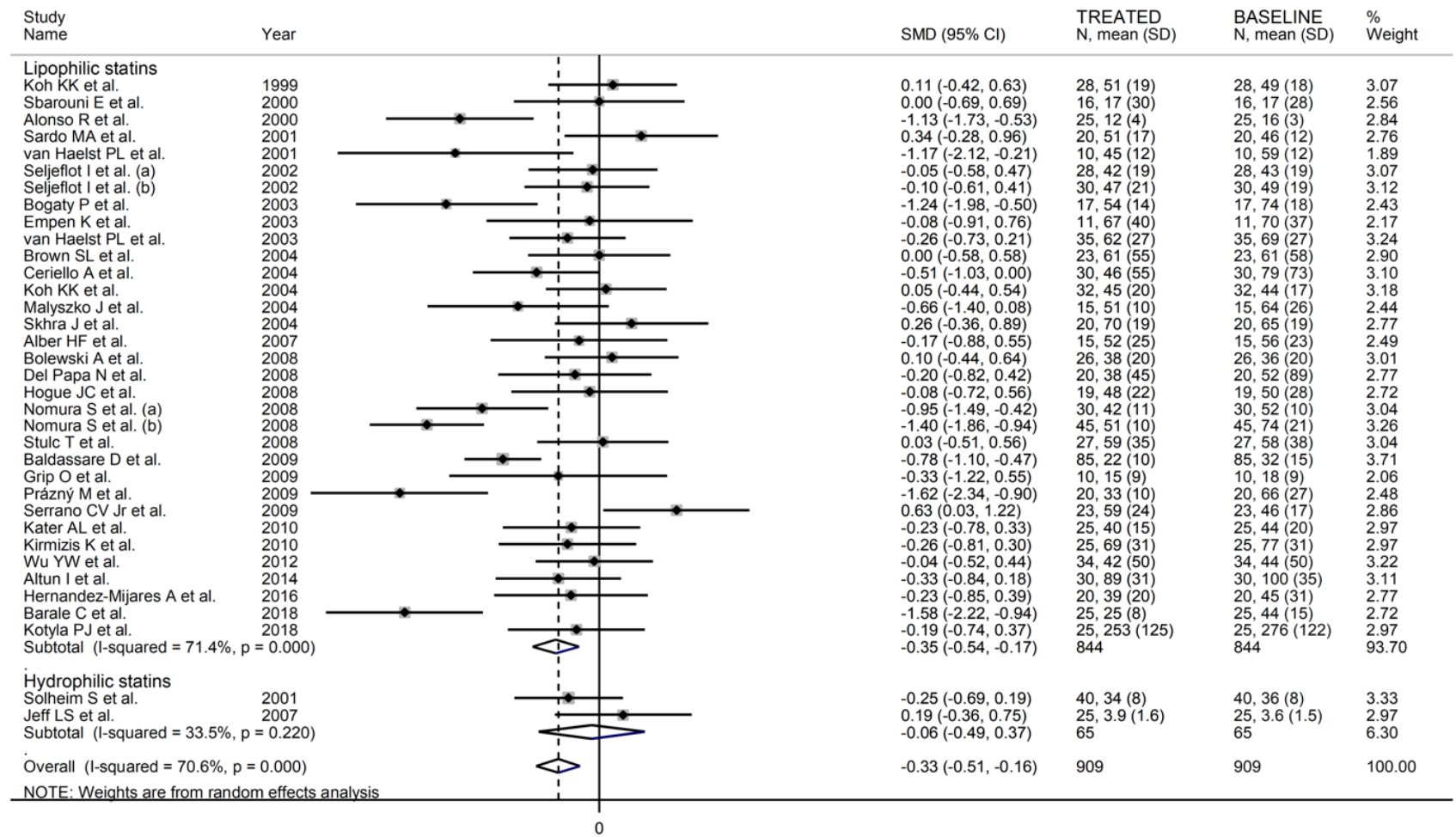

Figure 14. Forest plot of E-Selectin concentrations before and after statin treatment according to statin class (hydrophilic or lipophilic). 


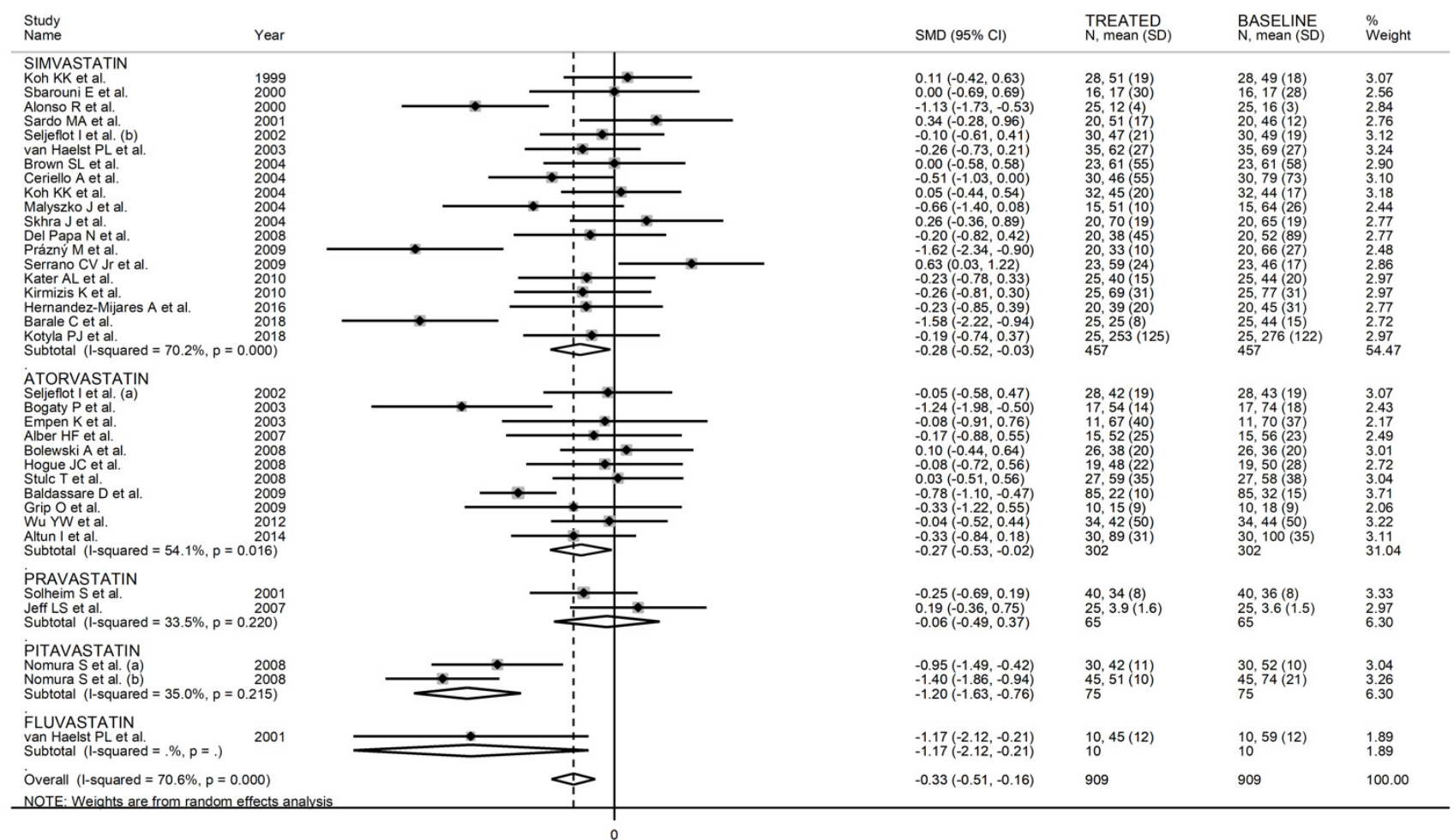

Figure 15. Forest plot of E-Selectin concentrations before and after statin treatment according to individual agents.

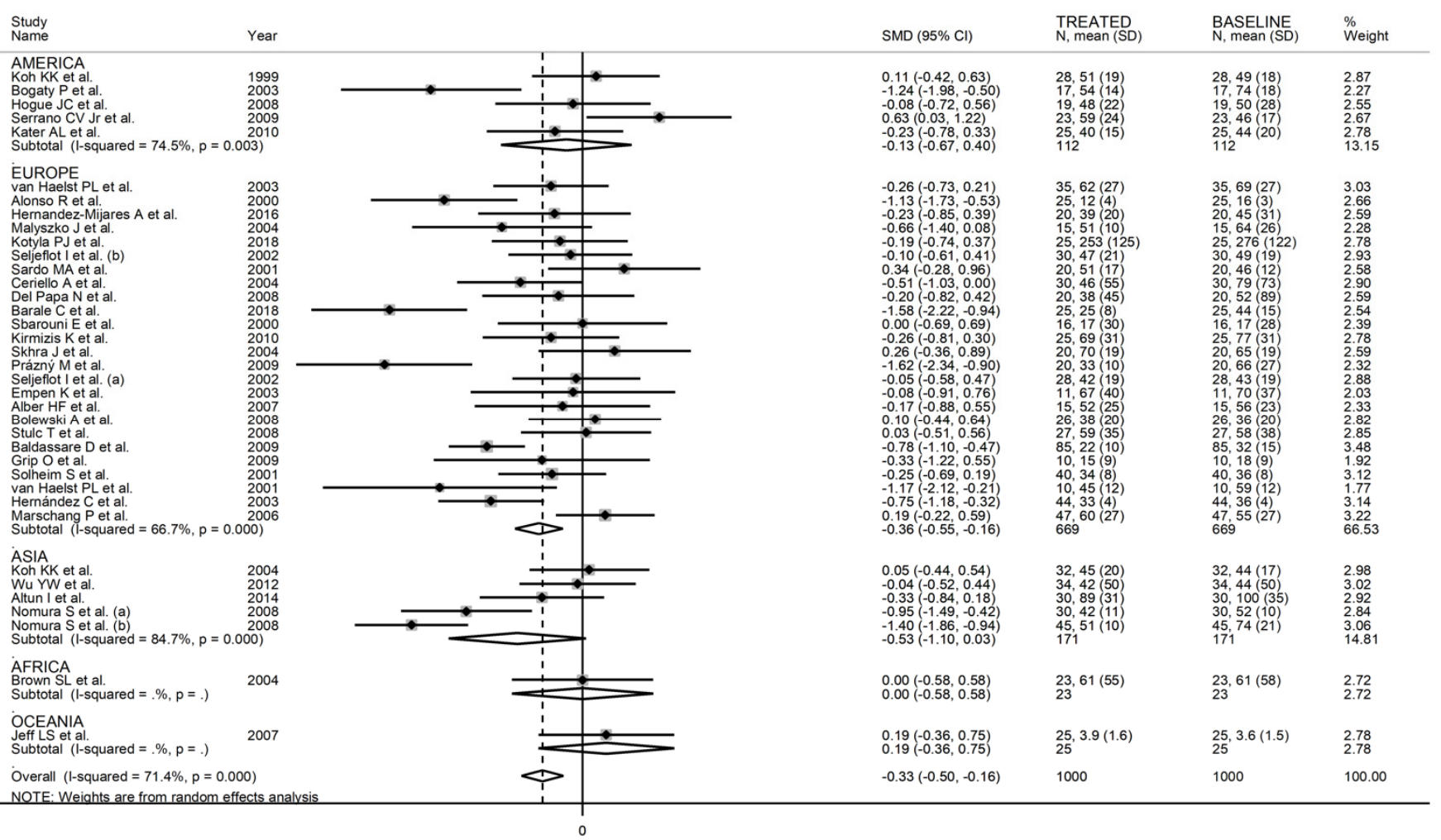

Figure 16. Forest plot of E-Selectin concentrations before and after statin treatment according to the continent where the study was conducted. 


\section{Discussion}

In this systematic review and meta-analysis, statin treatment significantly reduced the concentrations of soluble E-Selectin, L-Selectin, and P-Selectin in participants with a range of cardiovascular risk profiles. In sensitivity analysis, the pooled SMD values were not substantially altered when individual studies were sequentially removed. In meta-regression, no significant associations were observed between effect size and various patient and study characteristics, including baseline lipids. The absence of significant associations with treatment duration, ranging between 2 and 52 weeks, suggests that the selectin-lowering effects of statins are evident relatively early during treatment and are maintained for up to 1 year.

The activation of selectins, particularly P-Selectin and E-Selectin, has an established role in the pathogenesis of atherosclerosis and its clinical manifestations [94]. The results of several observational studies further support this proposition. In the Women's Health Study, participants with baseline soluble P-selectin concentrations in the highest quartile had a relative risk of suffering a cardiovascular event during a 3.5-year follow up period 2.2 times higher than those in the lowest quartile (95\% CI 1.2 to 4.2 ). Notably, this association was independent of obesity, hypertension, hypercholesterolaemia, diabetes, and physical activity [8]. In another study in 733 patients undergoing coronary revascularization, those with baseline P-selectin concentrations in the second, third, and fourth quartile were at higher risk of experiencing a major cardiovascular event during a 9.7-year follow up period compared to the first quartile (hazard ratio, HR, 1.23, 95\% CI 0.90 to 1.69; HR $1.48,95 \%$ CI 1.08 to 2.02 ; and HR 1.57, 95\% CI 1.11 to 2.15, respectively), after adjusting for confounders [9]. In a prospective study of 1041 adult patients with end-stage chronic kidney disease, each 0.1-log unit increase in P-selectin concentrations was significantly associated, after adjusting for confounders, with cardiovascular mortality (HR 1.10, 95\% CI 1.02 to 1.27) and sudden cardiac death (HR 1.12, 95\% CI 1.01 to 1.25 ) in males, but not in females, during a median follow-up of 38.2 months [10]. In a similar group of patients with end-stage renal disease, the risk of fatal and non-fatal cardiovascular events was significantly higher, after adjusting for confounders, in the highest vs. lowest tertile of soluble E-Selectin concentrations (HR 1.93, 95\% CI 1.03 to 3.56) during a 21-month follow-up period [11]. Finally, in 423 patients with non-valvular atrial fibrillation and other cardiovascular risk factors followed for 19 months, those with soluble E-Selectin concentrations in the upper tertile had a significantly higher risk of adverse clinical events when compared to the bottom tertile (relative risk, RR, 3.70, 95\% CI 2.51 to 5.31) [12].

The observed associations between soluble selectins and cardiovascular risk have stimulated the search for novel therapies that target these cell adhesion molecules. One of these agents, the monoclonal antibody against P-selectin inclacumab, has shown some promise in minimizing cardiac damage in patients with acute coronary syndrome undergoing percutaneous coronary intervention $[95,96]$. The results of our systematic review and meta-analysis suggest that soluble selectin-lowering might also be important in the context of statin therapy, an established treatment option in primary and secondary cardiovascular prevention [97-99]. Whist the exact mechanisms of action involved in the statin-mediated reduction in soluble selectin concentrations remain elusive, in vitro studies have shown that atorvastatin significantly prevents the overexpression of E-Selectin induced by cigarette smoking extract in human umbilical vein endothelial cells through inhibiting the $\mathrm{NF}_{-\mathrm{K}} \mathrm{B}$ signal pathway, a critical pathway involved in inflammatory processes [100,101]. Treatment with simvastatin has been shown to prevent the release of the enzyme semicarbazidesensitive amine oxidase/vascular adhesion protein 1, with consequent reduction of soluble E-Selectin [102]. Similar effects of simvastatin on the expression of P-Selectin and E-selectin have been reported in other studies [103]. Furthermore, treatment with atorvastatin significantly reduced the expression of P-Selectin in platelet-derived microparticles in patients with peripheral vascular disease [104].

In subgroup analysis, lipophilic, but not hydrophilic, statins significantly reduced soluble E-Selectin concentrations. However, these results need to be interpreted with cau- 
tion because of the extremely low number of treatment arms, two, involving hydrophilic statins. Significant differences were also observed with individual statins, with simvastatin and fluvastatin being particularly effective against P-Selectin, and simvastatin, atorvastatin, and pitavastatin against E-Selectin. Further research is warranted to investigate whether specific statin classes and individual agents have superior capacity to reduce soluble selectin concentrations and whether this effect might be particularly beneficial in specific patient groups. Another interesting observation, in subgroup analysis, was the difference in selectin-lowering according to specific continent, with studies conducted in Europe showing a particular efficacy against P-Selectin and E-Selectin. Previous studies have investigated the concentrations of soluble selectins in different ethnic groups. In the Multi-Ethnic Study of Atherosclerosis, no significant differences in soluble E-Selectin concentrations were observed between white, black, Hispanic, and Chinese participants [105]. Other studies have also failed to detect significant differences in soluble E-Selectin and P-Selectin across ethnic groups [106-108]. It remains to be established whether potential ethnic-related differences in statin-mediated selectin-lowering effects might translate into different effects on surrogate markers and/or clinical endpoints in intervention trials.

The strengths of our study include the relatively large number of treatment arms analysed (41 for P-Selectin, five for L-Selectin, and 41 for E-Selectin), the assessment of possible associations between effect size and a comprehensive range of study and patient characteristics by means of meta-regression and/or subgroup analysis, and a robust assessment of the certainty of evidence according to GRADE. One significant limitation is the large-to-extreme between-study heterogeneity, which limits the generalizability of our results. However, particularly in studies investigating P-selectin, this heterogeneity was substantially attenuated in a sub-group of studies performed in Europe using atorvastatin.

\section{Conclusions}

In this systematic review and meta-analysis, treatment with statins was associated with a significant reduction in the concentrations of soluble P-Selectin, L-Selectin, and E-Selectin, a critical family of cell adhesion molecules that is involved in the pathogenesis of atherosclerosis. The selectin-lowering effect was independent of various patient and study characteristics, particularly baseline lipid profile and treatment duration, and was more prominent with specific agents, i.e., simvastatin, in studies conducted in Europe. These results warrant adequately designed intervention trials to determine whether selectinlowering can mediate the atheroprotective effects of these agents and whether specific patient groups are more likely to benefit from this phenomenon. In particular, the reported differences in effect size according to the continent where the study was conducted require further research to determine whether ethnicity is an important mediator of the effects of statin treatment on circulating soluble selectins.

Supplementary Materials: The following are available online at https:/ /www.mdpi.com/article/10 .3390 /biomedicines9111707/s1, Table S1: PRISMA 2020 abstract checklist; Table S2: PRISMA 2020 checklist and search strategy.

Author Contributions: A.Z. and A.A.M. designed the study, screened the articles, assessed the risk of bias, extracted the data, analysed, and interpreted the data. A.A.M. wrote the first draft of the manuscript. A.A.M. and A.Z. reviewed the subsequent versions and the final draft. All authors have read and agreed to the published version of the manuscript.

Funding: This research received no external funding.

Institutional Review Board Statement: Not required as this was a systematic review and metaanalysis of published studies.

Informed Consent Statement: Not required as this was a systematic review and meta-analysis of published studies.

Data Availability Statement: The data that support the findings of this systematic review and meta-analysis are available from the first author, A.Z., upon reasonable request. 
Conflicts of Interest: The authors declare no conflict of interest.

\section{References}

1. Price, D.T.; Loscalzo, J. Cellular adhesion molecules and atherogenesis. Am. J. Med. 1999, 107, 85-97. [CrossRef]

2. McEver, R.P. Selectins: Lectins that initiate cell adhesion under flow. Curr. Opin. Cell Biol. 2002, 14, 581-586. [CrossRef]

3. Eriksson, E.E.; Xie, X.; Werr, J.; Thoren, P.; Lindbom, L. Importance of primary capture and L-selectin-dependent secondary capture in leukocyte accumulation in inflammation and atherosclerosis in vivo. J. Exp. Med. 2001, 194, 205-218. [CrossRef] [PubMed]

4. Galkina, E.; Ley, K. Vascular adhesion molecules in atherosclerosis. Arterioscler. Thromb. Vasc. Biol. 2007, 27, 2292-2301. [CrossRef]

5. Roldan, V.; Marin, F.; Lip, G.Y.; Blann, A.D. Soluble E-selectin in cardiovascular disease and its risk factors. A review of the literature. Thromb. Haemost. 2003, 90, 1007-1020. [CrossRef]

6. Nagy, B., Jr.; Miszti-Blasius, K.; Kerenyi, A.; Clemetson, K.J.; Kappelmayer, J. Potential therapeutic targeting of platelet-mediated cellular interactions in atherosclerosis and inflammation. Curr. Med. Chem. 2012, 19, 518-531. [CrossRef]

7. Theofilis, P.; Sagris, M.; Oikonomou, E.; Antonopoulos, A.S.; Siasos, G.; Tsioufis, C.; Tousoulis, D. Inflammatory Mechanisms Contributing to Endothelial Dysfunction. Biomedicines 2021, 9, 781. [CrossRef]

8. Ridker, P.M.; Buring, J.E.; Rifai, N. Soluble P-selectin and the risk of future cardiovascular events. Circulation 2001, 103, 491-495. [CrossRef]

9. Tscharre, M.; Vogel, B.; Tentzeris, I.; Freynhofer, M.K.; Rohla, M.; Wojta, J.; Weiss, T.W.; Ay, C.; Huber, K.; Farhan, S. Prognostic Impact of Soluble P-Selectin on Long-Term Adverse Cardiovascular Outcomes in Patients Undergoing Percutaneous Coronary Intervention. Thromb. Haemost. 2019, 119, 340-347. [CrossRef]

10. Scialla, J.J.; Plantinga, L.C.; Kao, W.H.; Jaar, B.; Powe, N.R.; Parekh, R.S. Soluble P-selectin levels are associated with cardiovascular mortality and sudden cardiac death in male dialysis patients. Am. J. Nephrol. 2011, 33, 224-230. [CrossRef]

11. Malatino, L.S.; Stancanelli, B.; Cataliotti, A.; Bellanuova, I.; Fatuzzo, P.; Rapisarda, F.; Leonardis, D.; Tripepi, G.; Mallamaci, F.; Zoccali, C. Circulating E-selectin as a risk marker in patients with end-stage renal disease. J. Intern. Med. 2007, 262, $479-487$. [CrossRef]

12. Krishnamoorthy, S.; Khoo, C.W.; Lim, H.S.; Lane, D.A.; Pignatelli, P.; Basili, S.; Violi, F.; Lip, G.Y. Prognostic role of plasma von Willebrand factor and soluble E-selectin levels for future cardiovascular events in a 'real-world' community cohort of patients with atrial fibrillation. Eur. J. Clin. Investig. 2013, 43, 1032-1038. [CrossRef]

13. Voutyritsa, E.; Kyriakos, G.; Patsouras, A.; Damaskos, C.; Garmpi, A.; Diamantis, E.; Garmpis, N.; Savvanis, S. Experimental Agents for the Treatment of Atherosclerosis: New Directions. J. Exp. Pharmacol. 2021, 13, 161-179. [CrossRef]

14. Chou, R.; Dana, T.; Blazina, I.; Daeges, M.; Jeanne, T.L. Statins for Prevention of Cardiovascular Disease in Adults: Evidence Report and Systematic Review for the US Preventive Services Task Force. JAMA 2016, 316, 2008-2024. [CrossRef]

15. Koskinas, K.C.; Siontis, G.C.M.; Piccolo, R.; Mavridis, D.; Raber, L.; Mach, F.; Windecker, S. Effect of statins and non-statin LDL-lowering medications on cardiovascular outcomes in secondary prevention: A meta-analysis of randomized trials. Eur. Heart J. 2018, 39, 1172-1180. [CrossRef]

16. Liao, J.K. Effects of statins on 3-hydroxy-3-methylglutaryl coenzyme a reductase inhibition beyond low-density lipoprotein cholesterol. Am. J. Cardiol. 2005, 96, 24F-33F. [CrossRef]

17. Oikonomou, E.; Leopoulou, M.; Theofilis, P.; Antonopoulos, A.S.; Siasos, G.; Latsios, G.; Mystakidi, V.C.; Antoniades, C.; Tousoulis, D. A link between inflammation and thrombosis in atherosclerotic cardiovascular diseases: Clinical and therapeutic implications. Atherosclerosis 2020, 309, 16-26. [CrossRef]

18. Wong, D.; Dorovini-Zis, K. Regualtion by cytokines and lipopolysaccharide of E-selectin expression by human brain microvessel endothelial cells in primary culture. J. Neuropathol. Exp. Neurol. 1996, 55, 225-235. [CrossRef]

19. Hansson, G.K. Immune mechanisms in atherosclerosis. Arterioscler. Thromb. Vasc. Biol. 2001, 21, 1876-1890. [CrossRef]

20. Moola, S.; Munn, Z.; Tufanaru, C.; Aromataris, E.; Sears, K.; Sfetcu, R.; Currie, M.; Qureshi, R.; Mattis, P.; Lisy, K.; et al. Systematic reviews of etiology and risk. In Joanna Briggs Institute Reviewer's Manual; Aromataris, E., Munn, Z., Eds.; Johanna Briggs Institute: Adelaide, SA, Australia, 2017.

21. Cohen, J. Statistical Power Analysis for the Behavioral Sciences, 2nd ed.; Erlbaum: Hillsdale, NJ, USA, 1988.

22. Balshem, H.; Helfand, M.; Schunemann, H.J.; Oxman, A.D.; Kunz, R.; Brozek, J.; Vist, G.E.; Falck-Ytter, Y.; Meerpohl, J.; Norris, S.; et al. GRADE guidelines: 3. Rating the quality of evidence. J. Clin. Epidemiol. 2011, 64, 401-406. [CrossRef]

23. Hultcrantz, M.; Rind, D.; Akl, E.A.; Treweek, S.; Mustafa, R.A.; Iorio, A.; Alper, B.S.; Meerpohl, J.J.; Murad, M.H.; Ansari, M.T.; et al. The GRADE Working Group clarifies the construct of certainty of evidence. J. Clin. Epidemiol. 2017, 87, 4-13. [CrossRef]

24. Zhang, Y.; Coello, P.A.; Guyatt, G.H.; Yepes-Nunez, J.J.; Akl, E.A.; Hazlewood, G.; Pardo-Hernandez, H.; Etxeandia-Ikobaltzeta, I.; Qaseem, A.; Williams, J.W., Jr.; et al. GRADE guidelines: 20. Assessing the certainty of evidence in the importance of outcomes or values and preferences-inconsistency, imprecision, and other domains. J. Clin. Epidemiol. 2019, 111, 83-93. [CrossRef]

25. Page, M.J.; McKenzie, J.E.; Bossuyt, P.M.; Boutron, I.; Hoffmann, T.C.; Mulrow, C.D.; Shamseer, L.; Tetzlaff, J.M.; Akl, E.A.; Brennan, S.E.; et al. The PRISMA 2020 statement: An updated guideline for reporting systematic reviews. BMJ 2021, 372, n71. [CrossRef]

26. Hozo, S.P.; Djulbegovic, B.; Hozo, I. Estimating the mean and variance from the median, range, and the size of a sample. BMC Med. Res. Methodol. 2005, 5, 13. [CrossRef] 
27. Bowden, J.; Tierney, J.F.; Copas, A.J.; Burdett, S. Quantifying, displaying and accounting for heterogeneity in the meta-analysis of RCTs using standard and generalised Q statistics. BMC Med. Res. Methodol. 2011, 11, 41. [CrossRef]

28. Higgins, J.P.; Thompson, S.G. Quantifying heterogeneity in a meta-analysis. Stat. Med. 2002, 21, 1539-1558. [CrossRef]

29. Tobias, A. Assessing the influence of a single study in the meta-analysis estimate. Stata Tech. Bull. 1999, 47, 15-17.

30. Begg, C.B.; Mazumdar, M. Operating characteristics of a rank correlation test for publication bias. Biometrics 1994, 50, $1088-1101$. [CrossRef]

31. Sterne, J.A.; Egger, M. Funnel plots for detecting bias in meta-analysis: Guidelines on choice of axis. J. Clin. Epidemiol. 2001, 54, 1046-1055. [CrossRef]

32. Duval, S.; Tweedie, R. Trim and fill: A simple funnel-plot-based method of testing and adjusting for publication bias in meta-analysis. Biometrics 2000, 56, 455-463. [CrossRef]

33. Koh, K.K.; Cardillo, C.; Bui, M.N.; Hathaway, L.; Csako, G.; Waclawiw, M.A.; Panza, J.A.; Cannon, R.O., 3rd. Vascular effects of estrogen and cholesterol-lowering therapies in hypercholesterolemic postmenopausal women. Circulation 1999, 99, 354-360. [CrossRef] [PubMed]

34. Rauch, U.; Osende, J.I.; Chesebro, J.H.; Fuster, V.; Vorchheimer, D.A.; Harris, K.; Harris, P.; Sandler, D.A.; Fallon, J.T.; Jayaraman, S.; et al. Statins and cardiovascular diseases: The multiple effects of lipid-lowering therapy by statins. Atherosclerosis 2000, 153, 181-189. [CrossRef]

35. Romano, M.; Mezzetti, A.; Marulli, C.; Ciabattoni, G.; Febo, F.; Di Ienno, S.; Roccaforte, S.; Vigneri, S.; Nubile, G.; Milani, M.; et al. Fluvastatin reduces soluble P-selectin and ICAM-1 levels in hypercholesterolemic patients: Role of nitric oxide. J. Investig. Med. 2000, 48, 183-189. [PubMed]

36. Sbarouni, E.; Kroupis, C.; Kyriakides, Z.S.; Koniavitou, K.; Kremastinos, D.T. Cell adhesion molecules in relation to simvastatin and hormone replacement therapy in coronary artery disease. Eur. Heart J. 2000, 21, 975-980. [CrossRef]

37. Alonso, R.; Mata, P.; De Andres, R.; Villacastin, B.P.; Martinez-Gonzalez, J.; Badimon, L. Sustained long-term improvement of arterial endothelial function in heterozygous familial hypercholesterolemia patients treated with simvastatin. Atherosclerosis 2001, 157, 423-429. [CrossRef]

38. Blann, A.D.; Gurney, D.; Hughes, E.; Buggins, P.; Silverman, S.H.; Lip, G.Y.H. Influence of Pravastatin on Lipoproteins, and on Endothelial, Platelet, and Inflammatory Markers in Subjects With Peripheral Artery Disease. Am. J. Cardiol. 2001, 88, 89-92. [CrossRef]

39. Sardo, M.A.; Castaldo, M.; Cinquegrani, M.; Bonaiuto, M.; Maesano, A.; Schepis, F.; Zema, M.C.; Campo, G.M.; Squadrito, F.; Saitta, A. Effects of simvastatin treatment on sICAM-1 and sE-selectin levels in hypercholesterolemic subjects. Atherosclerosis 2001, 155, 143-147. [CrossRef]

40. Solheim, S.; Seljeflot, I.; Arnesen, H.; Eritsland, J.; Eikvar, L. Reduced levels of TNF alpha in hypercholesterolemic individuals after treatment with pravastatin for 8 weeks. Atherosclerosis 2001, 157, 411-415. [CrossRef]

41. Van Haelst, P.L.; van Doormaal, J.J.; May, J.F.; Gans, R.O.; Crijns, H.J.; Tervaert, J.W. Secondary prevention with fluvastatin decreases levels of adhesion molecules, neopterin and C-reactive protein. Eur. J. Intern. Med. 2001, 12, 503-509. [CrossRef]

42. Atalar, E.; Ozmen, F.; Haznedaroglu, I.; Acil, T.; Ozer, N.; Ovunc, K.; Aksoyek, S.; Kes, S. Effects of short-term atorvastatin treatment on global fibrinolytic capacity, and sL-selectin and sFas levels in hyperlipidemic patients with coronary artery disease. Int. J. Cardiol. 2002, 84, 227-231. [CrossRef]

43. Seljeflot, I.; Tonstad, S.; Hjermann, I.; Arnesen, H. Reduced expression of endothelial cell markers after 1 year treatment with simvastatin and atorvastatin in patients with coronary heart disease. Atherosclerosis 2002, 162, 179-185. [CrossRef]

44. Bogaty, P.; Dagenais, G.R.; Poirier, P.; Boyer, L.; Auclair, L.; Pepin, G.; Jobin, J.; Arsenault, M. Effect of atorvastatin on exerciseinduced myocardial ischemia in patients with stable angina pectoris. Am. J. Cardiol. 2003, 92, 1192-1195. [CrossRef]

45. Dalla Nora, E.; Passaro, A.; Zamboni, P.F.; Calzoni, F.; Fellin, R.; Solini, A. Atorvastatin improves metabolic control and endothelial function in type 2 diabetic patients: A placebo-controlled study. J. Endocrinol. Investig. 2003, 26, 73-78. [CrossRef]

46. Empen, K.; Frost, R.J.; Geiss, H.C.; Otto, C.; Parhofer, K.G. Differential effects of fenofibrate versus atorvastatin on the concentrations of E-selectin and vascular cellular adhesion molecule- 1 in patients with type 2 diabetes mellitus and mixed hyperlipoproteinemia: A randomized cross-over trial. Cardiovasc. Diabetol. 2003, 2, 17. [CrossRef]

47. Ferroni, P.; Martini, F.; Cardarello, C.M.; Gazzaniga, P.P.; Davi, G.; Basili, S. Enhanced interleukin-1beta in hypercholesterolemia: Effects of simvastatin and low-dose aspirin. Circulation 2003, 108, 1673-1675. [CrossRef]

48. Hernandez, C.; Lecube, A.; Barbera, G.; Chacon, P.; Lima, J.; Simo, R. Effects of hypolipidemic treatment on serum markers of vascular inflammation in dyslipidemic men. Med. Sci. Monit. 2003, 9, CR114-CR119.

49. Homma, Y.; Homma, K.; Iizuka, S.; Iigaya, K. Effects of Fluvastatin on Plasma Levels of Low-Density Lipoprotein Subfractions, Oxidized Low-Density Lipoprotein, and Soluble Adhesion Molecules: A Twenty-Four-Week, Open-Label, Dose-Increasing Study. Curr. Ther. Res. 2003, 64, 236-247. [CrossRef]

50. Malyszko, J.; Malyszko, J.S.; Mysliwiec, M. Fluvastin therapy affects TAFI concentration in kidney transplant recipients. Transpl. Int. 2003, 16, 53-57. [CrossRef]

51. Nawawi, H.; Osman, N.S.; Annuar, R.; Khalid, B.A.K.; Yusoff, K. Soluble intercellular adhesion molecule-1 and interleukin-6 levels reflect endothelial dysfunction in patients with primary hypercholesterolaemia treated with atorvastatin. Atherosclerosis 2003, 169, 283-291. [CrossRef] 
52. Puccetti, L.; Pasqui, A.L.; Pastorelli, M.; Bova, G.; Di Renzo, M.; Leo, A.; Cercignani, M.; Palazzuoli, A.; Auteri, A.; Bruni, F. Platelet hyperactivity after statin treatment discontinuation. Thromb. Haemost. 2003, 90, 476-482. [CrossRef]

53. Van Haelst, P.L.; van Doormaal, J.J.; Asselbergs, F.W.; van Roon, A.M.; Veeger, N.J.; Henneman, M.M.; Smit, A.J.; Tervaert, J.W.; May, J.F.; Gans, R.O. Correlates of endothelial function and their relationship with inflammation in patients with familial hypercholesterolaemia. Clin. Sci. 2003, 104, 627-632. [CrossRef]

54. Brown, S.L.; Raal, F.J.; Panz, V.R.; Stevens, B.A.; Veller, M.G. High-dose atorvastatin therapy is required for significant improvement of endothelial function in heterozygous familial hypercholesterolaemic patients. Cardiovasc. J. S. Afr. 2004, 15, 70-75.

55. Ceriello, A.; Quagliaro, L.; Piconi, L.; Assaloni, R.; Da Ros, R.; Maier, A.; Esposito, K.; Giugliano, D. Effect of postprandial hypertriglyceridemia and hyperglycemia on circulating adhesion molecules and oxidative stress generation and the possible role of simvastatin treatment. Diabetes 2004, 53, 701-710. [CrossRef]

56. Cha, J.K.; Jeong, M.H.; Kim, J.W. Statin reduces the platelet P-selectin expression in atherosclerotic ischemic stroke. J. Thromb. Thrombolysis 2004, 18, 39-42. [CrossRef]

57. Koh, K.K.; Son, J.W.; Ahn, J.Y.; Jin, D.K.; Kim, H.S.; Choi, Y.M.; Ahn, T.H.; Kim, D.S.; Shin, E.K. Vascular effects of diet and statin in hypercholesterolemic patients. Int. J. Cardiol. 2004, 95, 185-191. [CrossRef]

58. Malyszko, J.; Malyszko, J.S.; Hryszko, T.; Mysliwiec, M. Increased soluble CD40L levels are reduced by long-term simvastatin treatment in peritoneally dialyzed patients. Blood Coagul. Fibrinolysis 2004, 15, 463-467. [CrossRef]

59. Nomura, S.; Shouzu, A.; Omoto, S.; Nishikawa, M.; Iwasaka, T. Effects of losartan and simvastatin on monocyte-derived microparticles in hypertensive patients with and without type 2 diabetes mellitus. Clin. Appl. Thromb. Hemost. 2004, 10, 133-141. [CrossRef]

60. Skrha, J.; Stulc, T.; Hilgertova, J.; Weiserova, H.; Kvasnicka, J.; Ceska, R. Effect of simvastatin and fenofibrate on endothelium in Type 2 diabetes. Eur. J. Pharmacol. 2004, 493, 183-189. [CrossRef]

61. Akcay, M.N.; Akcay, G.; Kiziltunc, A.; Ozturk, G.; Aydinli, B. The effect of short-term treatment with atorvastatin on E-selectin levels in severely burned patients. Int. J. Clin. Pharmacol. Res. 2005, 25, 65-69.

62. Undas, A.; Celinska-Lowenhoff, M.; Domagala, T.B.; Iwaniec, T.; Dropinski, J.; Lowenhoff, T.; Szczeklik, A. Early antithrombotic and anti-inflammatory effects of simvastatin versus fenofibrate in patients with hypercholesterolemia. Thromb. Haemost. 2005, 94, 193-199. [CrossRef]

63. Bleske, B.E.; Nicklas, J.M.; Bard, R.L.; Brook, R.D.; Gurbel, P.A.; Bliden, K.P.; Rajagopalan, S.; Pitt, B. Neutral effect on markers of heart failure, inflammation, endothelial activation and function, and vagal tone after high-dose HMG-CoA reductase inhibition in non-diabetic patients with non-ischemic cardiomyopathy and average low-density lipoprotein level. J. Am. Coll. Cardiol. 2006, 47, 338-341. [CrossRef] [PubMed]

64. Marschang, P.; Friedrich, G.J.; Ditlbacher, H.; Stoeger, A.; Nedden, D.Z.; Kirchmair, R.; Dienstl, A.; Pachinger, O.; Patsch, J.R. Reduction of soluble P-selectin by statins is inversely correlated with the progression of coronary artery disease. Int. J. Cardiol. 2006, 106, 183-190. [CrossRef] [PubMed]

65. Peverill, R.E.; Smolich, J.J.; Malan, E.; Goldstat, R.; Davis, S.R. Comparison of effects of pravastatin and hormone therapy on soluble P-selectin and platelet P-selectin expression in postmenopausal hypercholesterolemic women. Maturitas 2006, 53, 158-165. [CrossRef]

66. Potaczek, D.P.; Undas, A.; Celinska-Lowenhoff, M.; Szczeklik, A. Interleukin-6 -174 G/C promoter polymorphism and effects of fenofibrate and simvastatin on inflammatory markers in hypercholesterolemic patients. Blood Coagul. Fibrinolysis 2006, 17, 35-38. [CrossRef] [PubMed]

67. Alber, H.F.; Frick, M.; Sussenbacher, A.; Dorler, J.; Dichtl, W.; Stocker, E.M.; Pachinger, O.; Weidinger, F. Effect of atorvastatin on peripheral endothelial function and systemic inflammatory markers in patients with stable coronary artery disease. Wien. Med. Wochenschr. 2007, 157, 73-78. [CrossRef] [PubMed]

68. Inami, N.; Nomura, S.; Shouzu, A.; Omoto, S.; Kimura, Y.; Takahashi, N.; Tanaka, A.; Nanba, M.; Shouda, Y.; Iwasaka, T. Effects of pitavastatin on adiponectin in patients with hyperlipidemia. Pathophysiol. Haemost. Thromb. 2007, 36, 1-8. [CrossRef] [PubMed]

69. Jeffs, L.S.; Skilton, F.; Nitschke, J.; Bannister, K.M.; Faull, R.J. Effect of pravastatin on markers of endothelial activation in dialysis patients. Nephrology 2007, 12, 234-238. [CrossRef]

70. Souza-Costa, D.C.; Sandrim, V.C.; Lopes, L.F.; Gerlach, R.F.; Rego, E.M.; Tanus-Santos, J.E. Anti-inflammatory effects of atorvastatin: Modulation by the T-786C polymorphism in the endothelial nitric oxide synthase gene. Atherosclerosis 2007, 193, 438-444. [CrossRef]

71. Barreto, A.C.; Maeda, N.Y.; Soares, R.P.; Cicero, C.; Lopes, A.A. Rosuvastatin and vascular dysfunction markers in pulmonary arterial hypertension: A placebo-controlled study. Braz. J. Med. Biol. Res. 2008, 41, 657-663. [CrossRef]

72. Bolewski, A.; Lipiecki, J.; Plewa, R.; Burchardt, P.; Siminiak, T. The effect of atorvastatin treatment on lipid profile and adhesion molecule levels in hypercholesterolemic patients: Relation to low-density lipoprotein receptor gene polymorphism. Cardiology 2008, 111, 140-146. [CrossRef]

73. Del Papa, N.; Cortiana, M.; Vitali, C.; Silvestris, I.; Maglione, W.; Comina, D.P.; Lucchi, T.; Cortelezzi, A. Simvastatin reduces endothelial activation and damage but is partially ineffective in inducing endothelial repair in systemic sclerosis. J. Rheumatol. 2008, 35, 1323-1328. 
74. Forst, T.; Wilhelm, B.; Pfutzner, A.; Fuchs, W.; Lehmann, U.; Schaper, F.; Weber, M.; Muller, J.; Konrad, T.; Hanefeld, M. Investigation of the vascular and pleiotropic effects of atorvastatin and pioglitazone in a population at high cardiovascular risk. Diabetes Vasc. Dis. Res. 2008, 5, 298-303. [CrossRef]

75. Hogue, J.C.; Lamarche, B.; Tremblay, A.J.; Bergeron, J.; Gagne, C.; Couture, P. Differential effect of atorvastatin and fenofibrate on plasma oxidized low-density lipoprotein, inflammation markers, and cell adhesion molecules in patients with type 2 diabetes mellitus. Metabolism 2008, 57, 380-386. [CrossRef]

76. Nomura, S.; Shouzu, A.; Omoto, S.; Inami, N.; Tanaka, A.; Nanba, M.; Shouda, Y.; Takahashi, N.; Kimura, Y.; Iwasaka, T. Correlation between adiponectin and reduction of cell adhesion molecules after pitavastatin treatment in hyperlipidemic patients with type 2 diabetes mellitus. Thromb. Res. 2008, 122, 39-45. [CrossRef]

77. Stulc, T.; Vrablik, M.; Kasalova, Z.; Marinov, I.; Svobodova, H.; Ceska, R. Leukocyte and endothelial adhesion molecules in patients with hypercholesterolemia: The effect of atorvastatin treatment. Physiol. Res. 2008, 57, 185-194. [CrossRef]

78. Wang, L.; Rockwood, J.; Zak, D.; Devaraj, S.; Jialal, I. Simvastatin reduces circulating plasminogen activator inhibitor 1 activity in volunteers with the metabolic syndrome. Metab. Syndr. Relat. Disord. 2008, 6, 149-152. [CrossRef]

79. Baldassarre, D.; Porta, B.; Camera, M.; Amato, M.; Arquati, M.; Brusoni, B.; Fiorentini, C.; Montorsi, P.; Romano, S.; Veglia, F.; et al. Markers of inflammation, thrombosis and endothelial activation correlate with carotid IMT regression in stable coronary disease after atorvastatin treatment. Nutr. Metab. Cardiovasc. Dis. 2009, 19, 481-490. [CrossRef]

80. Grip, O.; Janciauskiene, S. Atorvastatin reduces plasma levels of chemokine (CXCL10) in patients with Crohn's disease. PLoS ONE 2009, 4, e5263. [CrossRef]

81. Nomura, S.; Shouzu, A.; Omoto, S.; Inami, N.; Shimazu, T.; Satoh, D.; Kajiura, T.; Yamada, K.; Urase, F.; Maeda, Y.; et al. Effects of pitavastatin on monocyte chemoattractant protein-1 in hyperlipidemic patients. Blood Coagul. Fibrinolysis 2009, $20,440-447$. [CrossRef]

82. Prazny, M.; Kasalova, Z.; Mazoch, J.; Kvasnicka, J.; Skrha, J. Microvascular Reactivity and Endothelial Function in Type 2 Diabetic Patients with Hyperlipidemia Treated with Simvastatin: 3-year Follow-up. Prague Med. Rep. 2009, 110, $290-300$.

83. Serrano, C.V., Jr.; Pesaro, A.E.; de Lemos, J.A.; Rached, F.; Segre, C.A.; Gomes, F.; Ribeiro, A.F.; Nicolau, J.C.; Yoshida, V.M.; Monteiro, H.P. Native LDL-cholesterol mediated monocyte adhesion molecule overexpression is blocked by simvastatin. Cardiovasc. Drugs Ther. 2009, 23, 215-220. [CrossRef] [PubMed]

84. Fichtenbaum, C.J.; Yeh, T.M.; Evans, S.R.; Aberg, J.A. Treatment with pravastatin and fenofibrate improves atherogenic lipid profiles but not inflammatory markers in ACTG 5087. J. Clin. Lipidol. 2010, 4, 279-287. [CrossRef] [PubMed]

85. Kater, A.L.; Batista, M.C.; Ferreira, S.R. Improved endothelial function with simvastatin but unchanged insulin sensitivity with simvastatin or ezetimibe. Metabolism 2010, 59, 921-926. [CrossRef] [PubMed]

86. Kirmizis, D.; Papagianni, A.; Dogrammatzi, F.; Skoura, L.; Belechri, A.M.; Alexopoulos, E.; Efstratiadis, G.; Memmos, D. Effects of simvastatin on markers of inflammation, oxidative stress and endothelial cell apoptosis in patients on chronic hemodialysis. J. Atheroscler. Thromb. 2010, 17, 1256-1265. [CrossRef]

87. Xu, D.Y.; Shu, J.; Huang, Q.Y.; Wasti, B.; Chen, C.; Liu, L.; Zhao, S.P. Evaluation of the lipid lowering ability, anti-inflammatory effects and clinical safety of intensive therapy with Zhibitai, a Chinese traditional medicine. Atherosclerosis 2010, 211, 237-241. [CrossRef]

88. Wu, Y.W.; Kao, H.L.; Huang, C.L.; Chen, M.F.; Lin, L.Y.; Wang, Y.C.; Lin, Y.H.; Lin, H.J.; Tzen, K.Y.; Yen, R.F.; et al. The effects of 3-month atorvastatin therapy on arterial inflammation, calcification, abdominal adipose tissue and circulating biomarkers. Eur. J. Nucl. Med. Mol. Imaging 2012, 39, 399-407. [CrossRef]

89. Altun, I.; Oz, F.; Arkaya, S.C.; Altun, I.; Bilge, A.K.; Umman, B.; Turkoglu, U.M. Effect of statins on endothelial function in patients with acute coronary syndrome: A prospective study using adhesion molecules and flow-mediated dilatation. J. Clin. Med. Res. 2014, 6, 354-361. [CrossRef]

90. Pawelczyk, M.; Chmielewski, H.; Kaczorowska, B.; Przybyla, M.; Baj, Z. The influence of statin therapy on platelet activity markers in hyperlipidemic patients after ischemic stroke. Arch. Med. Sci. 2015, 11, 115-121. [CrossRef]

91. Hernandez-Mijares, A.; Banuls, C.; Rovira-Llopis, S.; Diaz-Morales, N.; Escribano-Lopez, I.; de Pablo, C.; Alvarez, A.; Veses, S.; Rocha, M.; Victor, V.M. Effects of simvastatin, ezetimibe and simvastatin/ezetimibe on mitochondrial function and leukocyte/endothelial cell interactions in patients with hypercholesterolemia. Atherosclerosis 2016, 247, 40-47. [CrossRef]

92. Barale, C.; Frascaroli, C.; Senkeev, R.; Cavalot, F.; Russo, I. Simvastatin Effects on Inflammation and Platelet Activation Markers in Hypercholesterolemia. Biomed. Res. Int. 2018, 2018, 6508709. [CrossRef]

93. Kotyla, P.J. Short course of simvastatin has no effect on markers of endothelial activation in normolipidemic patients with systemic sclerosis. J. Int. Med. Res. 2018, 46, 1893-1901. [CrossRef]

94. Dong, Z.M.; Chapman, S.M.; Brown, A.A.; Frenette, P.S.; Hynes, R.O.; Wagner, D.D. The combined role of P- and E-selectins in atherosclerosis. J. Clin. Investig. 1998, 102, 145-152. [CrossRef]

95. Tardif, J.C.; Tanguay, J.F.; Wright, S.R.; Duchatelle, V.; Petroni, T.; Gregoire, J.C.; Ibrahim, R.; Heinonen, T.M.; Robb, S.; Bertrand, O.F.; et al. Effects of the P-selectin antagonist inclacumab on myocardial damage after percutaneous coronary intervention for non-ST-segment elevation myocardial infarction: Results of the SELECT-ACS trial. J. Am. Coll. Cardiol. 2013, 61, $2048-2055$. [CrossRef] 
96. Stahli, B.E.; Gebhard, C.; Duchatelle, V.; Cournoyer, D.; Petroni, T.; Tanguay, J.F.; Robb, S.; Mann, J.; Guertin, M.C.; Wright, R.S.; et al. Effects of the P-Selectin Antagonist Inclacumab on Myocardial Damage After Percutaneous Coronary Intervention According to Timing of Infusion: Insights From the SELECT-ACS Trial. J. Am. Heart Assoc. 2016, 5. [CrossRef]

97. Cai, T.; Abel, L.; Langford, O.; Monaghan, G.; Aronson, J.K.; Stevens, R.J.; Lay-Flurrie, S.; Koshiaris, C.; McManus, R.J.; Hobbs, F.D.R.; et al. Associations between statins and adverse events in primary prevention of cardiovascular disease: Systematic review with pairwise, network, and dose-response meta-analyses. BMJ 2021, 374, n1537. [CrossRef]

98. Blais, J.E.; Wei, Y.; Yap, K.K.W.; Alwafi, H.; Ma, T.T.; Brauer, R.; Lau, W.C.Y.; Man, K.K.C.; Siu, C.W.; Tan, K.C.B.; et al. Trends in lipid-modifying agent use in 83 countries. Atherosclerosis 2021, 328, 44-51. [CrossRef]

99. Salami, J.A.; Warraich, H.; Valero-Elizondo, J.; Spatz, E.S.; Desai, N.R.; Rana, J.S.; Virani, S.S.; Blankstein, R.; Khera, A.; Blaha, M.J.; et al. National Trends in Statin Use and Expenditures in the US Adult Population From 2002 to 2013: Insights From the Medical Expenditure Panel Survey. JAMA Cardiol. 2017, 2, 56-65. [CrossRef]

100. Wang, H.; Yin, J.; Guo, Y. Atorvastatin might resist tobacco smoking-induced endothelial inflammation through the inhibition of NF-kappaB signal pathway. Clin. Exp. Hypertens. 2019, 41,1-4. [CrossRef]

101. Liu, T.; Zhang, L.; Joo, D.; Sun, S.C. NF-kappaB signaling in inflammation. Signal Transduct. Target. Ther. 2017, 2. [CrossRef]

102. Sun, P.; Hernandez-Guillamon, M.; Campos-Martorell, M.; Simats, A.; Montaner, J.; Unzeta, M.; Sole, M. Simvastatin blocks soluble SSAO/VAP-1 release in experimental models of cerebral ischemia: Possible benefits for stroke-induced inflammation control. Biochim. Biophys. Acta Mol. Basis Dis. 2018, 1864, 542-553. [CrossRef]

103. Eccles, K.A.; Sowden, H.; Porter, K.E.; Parkin, S.M.; Homer-Vanniasinkam, S.; Graham, A.M. Simvastatin alters human endothelial cell adhesion molecule expression and inhibits leukocyte adhesion under flow. Atherosclerosis 2008, 200, 69-79. [CrossRef]

104. Mobarrez, F.; He, S.; Broijersen, A.; Wiklund, B.; Antovic, A.; Antovic, J.; Egberg, N.; Jorneskog, G.; Wallen, H. Atorvastatin reduces thrombin generation and expression of tissue factor, P-selectin and GPIIIa on platelet-derived microparticles in patients with peripheral arterial occlusive disease. Thromb. Haemost. 2011, 106, 344-352. [CrossRef]

105. Steffen, B.T.; Steffen, L.M.; Tracy, R.; Siscovick, D.; Jacobs, D.; Liu, K.; He, K.; Hanson, N.Q.; Nettleton, J.A.; Tsai, M.Y. Ethnicity, plasma phospholipid fatty acid composition and inflammatory/endothelial activation biomarkers in the Multi-Ethnic Study of Atherosclerosis (MESA). Eur. J. Clin. Nutr. 2012, 66, 600-605. [CrossRef]

106. Stepanikova, I.; Bateman, L.B.; Oates, G.R. Systemic Inflammation in Midlife: Race, Socioeconomic Status, and Perceived Discrimination. Am. J. Prev. Med. 2017, 52, S63-S76. [CrossRef]

107. Makin, A.J.; Chung, N.A.; Silverman, S.H.; Lip, G.Y. Thrombogenesis and endothelial damage/dysfunction in peripheral artery disease. Relationship to ethnicity and disease severity. Thromb. Res. 2003, 111, 221-226. [CrossRef]

108. Jaumdally, R.J.; Varma, C.; Blann, A.D.; Macfadyen, R.J.; Lip, G.Y. Indices of angiogenesis, platelet activation, and endothelial damage/dysfunction in relation to ethnicity and coronary artery disease: Differences in central versus peripheral levels. Ann. Med. 2007, 39, 628-633. [CrossRef] 